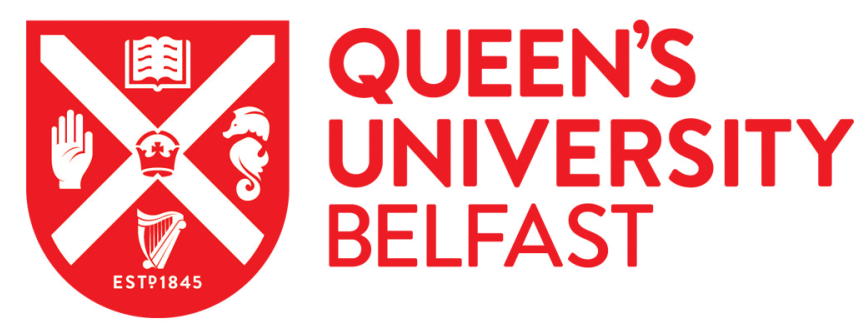

\title{
Adaptive d-Hop Connected Dominating Set in Highly Dynamic Flying Ad-hoc Networks
}

Duong, T. Q., \& Garcia-Palacios, E. (2021). Adaptive d-Hop Connected Dominating Set in Highly Dynamic Flying Ad-hoc Networks. IEEE Transactions on Network Science and Engineering, 8(3). https://doi.org/10.1109/TNSE.2021.3103873

\section{Published in:}

IEEE Transactions on Network Science and Engineering

\section{Document Version:}

Peer reviewed version

\section{Queen's University Belfast - Research Portal:}

Link to publication record in Queen's University Belfast Research Portal

\section{Publisher rights}

Copyright 2021 IEEE. This work is made available online in accordance with the publisher's policies. Please refer to any applicable terms of use of the publisher.

\section{General rights}

Copyright for the publications made accessible via the Queen's University Belfast Research Portal is retained by the author(s) and / or other copyright owners and it is a condition of accessing these publications that users recognise and abide by the legal requirements associated with these rights.

Take down policy

The Research Portal is Queen's institutional repository that provides access to Queen's research output. Every effort has been made to ensure that content in the Research Portal does not infringe any person's rights, or applicable UK laws. If you discover content in the Research Portal that you believe breaches copyright or violates any law, please contact openaccess@qub.ac.uk. 


\title{
Adaptive $d$-Hop Connected Dominating Set in Highly Dynamic Flying Ad-hoc Networks
}

\author{
Bowen Wang, Yanjing Sun, Member, IEEE, Tan Do-Duy, Emiliano Garcia-Palacios, Trung Q. Duong, Senior \\ Member, IEEE
}

\begin{abstract}
By exploring the intelligent cooperation of unmanned aerial vehicle (UAV) swarms, the formed flying adhoc networks (FANETs) can support a variety of collaborative operations with real-time communications in emergency scenarios. To reduce the prohibitively high routing overhead with the connectivity guaranteed of multi-hop links, UAV swarms can construct a virtual backbone network (VBN) based on the graph-theoretical $d$-hop connected dominating set ( $d$-CDS), where each UAV outside VBN can send collected data to VBN within $d$-hop distance. However, due to the high dynamics of FANETs in emergency scenarios, the optimal solution may not match the current status, which results in frequently intermittent connectivity. Besides, recomputing the solution from scratch will lead to significant maintance costs. Therefore, it is crucial to adapt the minimal $d$-CDS to topology changes. To this end, we propose an $\mathcal{O}(d \log (N))$-approximation algorithm (i.e., $N$ denotes the maximal number of nodes) with expected $\widetilde{\mathcal{O}}\left(d \Delta^{2}\right)$ (i.e., $\Delta$ denotes the maximal degree of a vertex over the sequence of updates) time per update. The simulation results demonstrate that our adaptive solution can strike a better tradeoff among the routing overhead, response time, and maintance costs per topology update compared with state-of-the-art schemes in emergency scenarios.
\end{abstract}

Index Terms-Flying ad-hoc networks, virtual backbone, dynamic topology structures, graph theory.

\section{INTRODUCTION}

$\mathbf{R}$ ECENTLY, unmanned aerial vehicles (UAVs) have gained significant attention for their application potential in cooperative and collaborative operation of dynamic missions in emergency scenarios [1]. By exploiting the swarm intelligence among UAVs and terminal-edge-cloud collaboration, a self-organized multi-UAV communication system termed as flying ad-hoc networks (FANETs) have emerged, which can be seen as a special case of mobile ad-hoc networks (MANETs)

This work was supported in part by the National Natural Science Foundation of China under Grant 62071472, in part by the the Program for "Industrial IoT and Emergency Collaboration " Innovative Research Team in China University of Mining and Technology (CUMT) under Grant 2020ZY002, in part by the Fundamental Research Foundation for the Central Universities under Grant 2020ZDPY0304, and in part by the Teaching Reform Research Project of the Ministry of Education for Teaching Steering Committee of the Electronic Information Specialty in Universities under Grant 2020-YB-54,.

Bowen Wang and Yanjing Sun are with the School of Information and Control Engineering, China University of Mining and Technology, Xuzhou, 221116, China (e-mail: \{bowenwang, yjsun\}@cumt.edu.cn). Bowen Wang and Yanjing Sun are also with the Xuzhou Engineering Research Center of Intelligent Industry Safety and Emergency Collaboration, Xuzhou, 221116, China.

Tan Do-Duy is with HCMC University of Technology and Education, Vietnam (email: tandd@hcmute.edu.vn)

Trung Q. Duong and Emiliano Garcia-Palacios are with the School of Electronics, Electrical Engineering and Computer Science, Queen's University Belfast, Belfast, BT7 1NN, UK (e-mail: trung.q.duong@qub.ac.uk; e.garcia@ee.qub.ac.uk). by replacing terrestrial devices with UAVs in the absence of fixed central infrastructure [2]-[4]. In a FANET, each flying node serves not only as a mobile host but also as a router at the same time [5]. Because of their advantages of flexible mobility, fast deployments, "bird's eye view" vision, selforganizing, and self-healing, FANETs have become particularly beneficial for resisting harsh environments, such as public security accidents and natural disasters [6]-[8]. In emergency situations, when terrestrial communication infrastructure or sensors are not available, a swarm of UAVs with sensing, computation, communication, caching, and control capabilities, can autonomously perform a mix of missions including situation sensing, data processing, connectivity enhancement, and communication recovery, etc. To coordinate rescue teams on the ground, flying nodes are remotely deployed to gather related information and then transmit it to the mobile ground station for further analysis.

In [6], Bekmezci et al. summarized four characteristics of FANETs. Firstly, due to the high degree of mobility, the topology structure of a FANET changes more frequently than that of the typical MANETs or vehicle ad hoc networks (VANETs). Secondly, due to the lack of ground infrastructure, a FANET has to support peer-to-peer communications for the purpose of swarm collaboration. Thirdly, the trajectory is restricted by collaborative features, and thus the mobility model of a FANET is more complicated. Fourthly, in extreme environments, each UAV node may move due to the obstacles avoidance and exhaustion of energy. Besides, due to the limited transmit power of UAVs, the direct communication link can only be built within a constrained communication range. By allowing considerable relaying operations, the multihop link through a series of intermediate nodes may incur a prohibitively high communication overhead. These characteristics result in frequently intermittent connectivity between UAVs while transmitting critical information, which poses huge challenges for the network routing and topology control in FANETs [9].

Generally, building a virtual backbone network (VBN) is widely used for simplifying the routing problem in MANETs and VANETs. The main idea of constructing a VBN is to reduce most routing and forwarding operations into partial nodes [10]. In [11], the VBN construction problem was formulated as a classic minimum connected dominating set (MCDS) problem, which was firsted introduced by [12] and has been widely used for effectively constructing a VBN in MANETs. A dominating set (DS) problem in graph theory is to find a subset $D$ in a given graph $G$ such that all the vertices are either in $D$ or adjacent to $D$ (i.e., dominated 
by $D$ ) [13]. A connected dominating set means that every two vertices in $D$ are connected. Thus, a MCDS based VBN can not only reduce the searching space for routing, but also ensure the connectivity [14]. The MCDS problem has been extensively studied in literature [11], [15]-[20]. From the algorithm design perspective, since the MCDS problem is NPComplete [21], the techniques for solving it can be classified into two main categories: heuristic algorithms [16], [18], [19] and approximation algorithms [11], [17], [20]. Compared with the fast heuristic algorithms, the approximation algorithms can provide theoretical guarantees with realistic complexity. From the perspective of graph structures, the simplified model can be classified into two main categories: geometric graphs such as unit desk graph (UDG) and unit ball graph(UBG) [17], [19], [20] and general graph [11], [16]. From the problem transformation perspective, the proposed methods are based on Maximum Independent Set (MIS) [16]-[18] and Minimum Spanning Tree (MST) [11], [15], [19], [20]. As the generalization of CDS, $d$-hop connected dominating set ( $d$-CDS) can balance the average path length (delay) and the size of CDS (maintenance cost) by assuming that each dominatee can be at most $d$-hop away from its dominator, in which $d$ can be flexibly set based on practical application scenario. In [22], Vuong et al. first proved that finding the minimal $d$-CDS is NP-complete. In [23], Azizian et al. proposed a heuristic algorithm for finding the minimal $d$-CDS and applied this algorithm for resource sharing in VANETs. In [24], Zhang et al. proposed an $\mathcal{O}\left(d^{2}\right)$-approximation algorithm to find a $d$ CDS in UDG. In [25], Gao et al. improved the approximation ratio into $2(d+1) \lambda$, where $\lambda$ denotes a parameter related to $d$ such that $\lambda \leq 18.4$. Most recently, Li et al. in [15] proposed a better $2(d+1) \beta$-approximation algorithm in three-dimentional space, i.e., UDG. However, the static approaches proposed by these works can only be applied to terrestrial ad-hoc networks whose topology structure is quasi-static or slightly dynamic.

Due to the stochasticity of extreme environments as well as the highly dynamic nature of FANETs, the backbone network in a FANET will be time-varying, which indicates that the current decision may not match the current topology. Besides, flying nodes may depart due to the low battery and rejoin when the recharing is finished, so the Air-to-Air (A2A) communication links will rapidly change over time and thus a fixed $d$-CDS cannot guarantee permanent connectivity. Hence, an effective $d$-CDS construction scheme requires that: 1) the $d$-CDS size is as small as possible to reduce the routing overhead while the delay is acceptable; 2) the $d$-CDS could adapt to topology changes with lower cost, which means that as few vertices as possible need to be replaced in a $d$-CDS for each topology change; 3) the update time is as short as possible. To mantain the topology connectivity characterized by the above mentioned issues, it is natural to design a smart approach which can adapt to the topology changes quickly. Because solving the whole problem from scratch will result in the delayed decision, which may not be matched to the status quo. Recently, more and more researchers have devoted to dynamic algorithms for solving several graph optimization problems, such as dynamic set cover (SC) [26], set multicover (SMC) [27], vertex cover [28], graph coloring [29], and
MIS [30]. In [31], the authors proposed a heuristic algorithms for $\mathrm{VBN}$ construction and maintenance in a FANET. More specifically, the main idea is to replace the current VBN with another CDS having the highest node similarity within all MCDS when topology changes. Notably, the selected MCDS may be invalid since its successful update rate is not $100 \%$.

Compared with heuristic algorithms, the approximation algorithms can provide the exact quantitative description about the gap between feasible solution and optimal solution with tractable complexity, which raises a natural question on how to design approximation algorithms for dynamic $d$-CDS. In [32], Leonidas et al. proposed an efficient algorithm to maintain a constant-factor approximation 1-CDS for dynamic geometric graph in cases of vertex insertions/deletions in general graph. Mapping to FANETs, solving the minimal $d$-CDS based on geometric graph means that all UAVs are considered to be homogeneous (i.e., they have similar functions and characteristics), which is not realistic since most FANETs have heterogeneity. Besides, both the algorithm design and theoretical optimality guarantees are subjected to the specific graph structure, which indicates that the proposed algorithms in [32] are not readily extended to solve the dynamic $d$-CDS problem over general graphs (corresponding to heterogeneous FANETs). In [33], Hjuler et al. designed algorithms that maintain a better $\mathcal{O}(\log (N))$ approximation under edge insertions/deletions for 1-CDS. However, the presented algorithm in [33] did not deal with the case of vertex insertions/deletions. To sum up, all these works studies are limited in terms of universal application such as the graph structure, the hop count $d$ (only applicable to the case $d=1$ ), and the types of topology changes, which inspires us to design an efficient algorithm adapting to all types of topology changes with arbitrary $d$ for all types of graphs.

To address all the aforementioned challenges, we first investigate how to dynamically update the $d$-CDS with lower cost based on a swarming-oriented mobility model, which lays a solid foundation for the dynamic $d$-CDS. Due to the frequently changing nature of FANETs, recomputing for each change is costly. As such, we consider an alternative approach by adaptively approximating the dynamic minimal $d$-CDS problem with $\mathcal{O}(d \log (N))$ approximation ratio that can deal with batches of changes. To ensure that the running time of maintaining the minimal $d$-CDS after each update is less than that of recomputing the whole problem from scratch, our proposed scheme only handles the part topology changes. The main contributions of this paper are listed as follows:

- To handle the topology changes of a FANET, we develop an adaptive solution framework for the stable VBN maintaince problem by dynamically restoring both the minimality and connectivity of a $d$-CDS with a batch of changes, including nodes entering, leaving and moving. As far as we know, this is the first work focusing on finding a dynamic $d$-CDS that adapts to all types of topology changes for all types of graphs.

- We propose an $\mathcal{O}(d \log (N))$-approximation algorithm (i.e., $N$ denotes the maximal number of nodes), which can adapt $d$-CDS to topology changes with expected $\widetilde{\mathcal{O}}\left(d \Delta^{2}\right)$ (i.e., $\Delta$ denotes the maximal degree of a vertex 


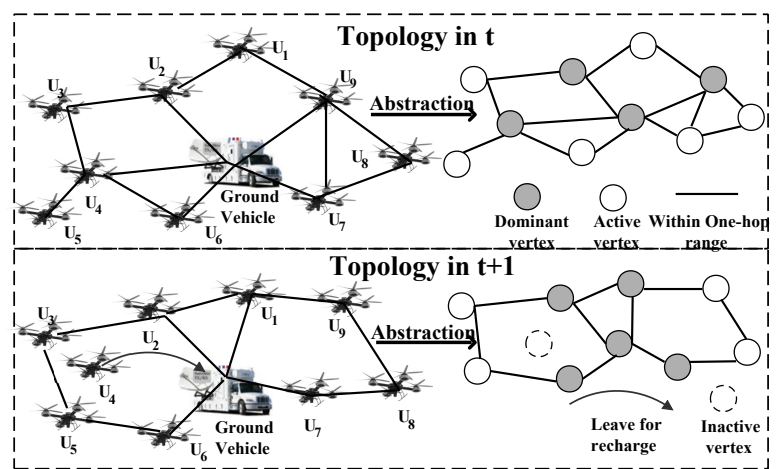

Fig. 1: Topology structures of a FANET in two consecutive time slots.

over the sequence of updates) time per vertex insertion/deletion ${ }^{1}$. As for the details of our algorithm, we first propose a hierarchical strategy to adaptively maintain the $\mathcal{O}(\log (N))$-approximation $d$-DS, and then dynamically repair the connectivity by maintaining a weighted MST and restore the minimality by maintaining a dynamic minimum spanning forest based on the top-tree data structure.

- Since finding the minimum $d$-CDS problem is NPcomplete, we comprehensively analyze the performance of our algorithm, including approximation ratio and time complexity. To verify the performance in a practical FANET scenario, we run extensive simulations based on a swarming-oriented mobility model. The simulation results show that the adaptive solution presented here can strike a better trade-off among the routing overhead, update time, and maintenance costs per topology update.

The rest of this paper is outlined as follows. The system models including mobility, energy, communication and networking models are described in Section II. Next, the dynamic VBN maintenance problem is presented and then the problem transformation is illustrated in Section III. The adaptive scheme to solve the dynamic minimal $d$-CDS problem is proposed and the performance analysis is discussed in Section IV. Simulation results are presented in Section V. Finally, conclusions are contained in Section VI.

\section{SySTEM MODEL}

As shown in Fig.1, we consider a typical FANET assisted by a mobile ground vehicle in an emergency scenario, where a set of multi-rotor UAVs are capable of providing sensing, data processing, and information services. More specifically, the crucial information is transmitted from UAVs to the ground vehicle $g$ for further analysis. Similar to [16] and [34], the ground vehicle can be considered as a ground control station and charging station. For simplicity, we assume that the ground vehicle is always in the topology center. Hereinafter, we will introduce the communication model, networking model, mobility model, and energy model.

${ }^{1}$ Note that $\widetilde{\mathcal{O}}\left(\Delta^{2}\right)$ here means $\mathcal{O}\left(\Delta^{2} \operatorname{polylog}(\Delta)\right.$, where polylog $(N)$ indicates the polynominal of $\log (N)$ [33]. In a word, the $\widetilde{\mathcal{O}}$ here can be viewed as $\mathcal{O}$ except ignoring logarithmic factors about $N$.

\section{A. Communication and Networking Models}

Different from most terrestrial wireless communication networks, UAV swarms are topology-dynamic, which makes neighboring cooperation quite necessary. For this purpose, local message exchange through $\mathrm{A} 2 \mathrm{~A}$ links is required. Besides, considering that the distance between edge UAVs and the destination, i.e., ground vehicle, may be beyond the transmission range of a single UAV, the multi-hop link can be constructed. Without loss of generality, we consider a time slotted system where the $\mathcal{T}$ is divided into $T$ sufficiently small time slots with equal length $\delta$. Thus, we use $\{1, \ldots, t, \ldots, T\}$ to represent the set of cumulative slots. In this case, the topology structure can be deemed unchanged in each time slot. Let $\mathcal{U}(t)=\left\{u_{i}\right\}_{i=1}^{N(t)}$ denote the UAV swarm, where $N(t)$ is the number of UAVs in slot $t$. To ensure that each node has a dedicated slot for broadcasting, time division multiple access (TDMA) can be applied and thus the interference among different links is neglected. In this paper, we mainly focus on the VBN construction and maintenance problem for topology control in dynamic environments.

For UAV $u_{i}$ and ground vehicle $g$, its real-time coordinates can be denoted as $\left(x_{i}^{u}(t), y_{i}^{u}(t), h_{i}^{u}(t)\right)$ and $\left(x^{g}(t), y^{g}(t), 0\right)$, respectively. Herein, the 3D Cartesian coordinate system is considered and the distance between two nodes can be calculated according to their Euclidean distance:

$$
d_{i, g}(t)=\sqrt{\left(x_{i}^{u}(t)-x^{g}(t)\right)^{2}+\left(y_{i}^{u}(t)-y^{g}(t)\right)+\left(h_{i}^{u}(t)\right)^{2}} .
$$

Without loss of generality, we consider a free-space path loss model in the A2A and Air-to-Ground (A2G) wireless channel dominated by line-of-sight (LOS) [35]. As such, the A2G links from the UAV to ground vehicle can experience LoS conditions. In slot $t$, the channel gain from the UAV $u_{i}$ to $u_{j}$ can be represented by

$$
g_{i, j}(t)=\eta_{0} d_{i, j}^{-\alpha}(t)
$$

where $\eta_{0}$ denotes the power gain of a LoS channel under unit reference distance, i.e., $d_{0}=1 \mathrm{~m}$, and $\alpha$ is the path-loss exponent. Note that the channel gain of A2A links can be calculated in the same way.

Given the transmit power $P_{i}^{t r}$ of $u_{i}$, the received signal-tonoise ratio $(\mathrm{SNR})$ at $u_{j}$ can be denoted as

$$
\gamma_{i, j}(t)=\frac{P_{i}^{t r} g_{i, j}(t)}{\sigma^{2}}
$$

where $\sigma^{2}$ denotes the white Gaussian noise variance. The A2A link is established successfully if $\gamma_{i, j}(t) \geq \gamma_{t h}$, where $\gamma_{t h}$ is a chosen SNR threshold. Hence, the maximum communication range for $u_{i}$ is represented by

$$
d_{i, j}(t) \leq d_{t h}^{i}=\left(\frac{\eta_{0} P_{i}^{t r}}{\sigma^{2} \gamma_{t h}}\right)^{1 / \alpha} .
$$

Equipped with an omnidirectional antenna, the communication range of each UAV can be denoted as a sphere with radius $R_{c}^{i}=d_{t h}^{i}$. In this case, each two nodes can build a communication link if and only if they are within the communication range of each other. In this way, we abstract the time-varying 


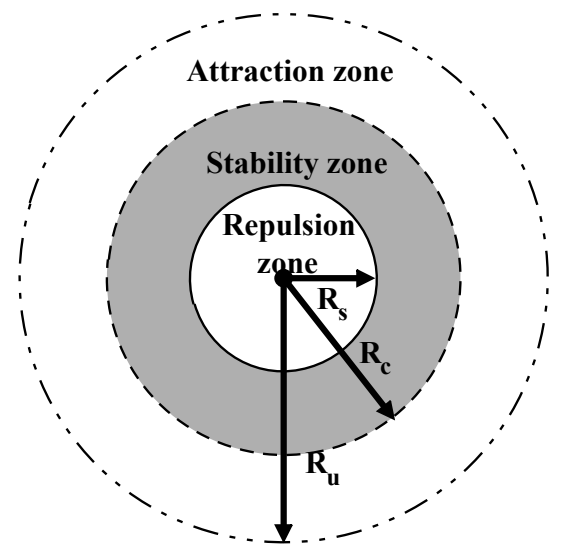

Fig. 2: Illustration of sensing zone structure.

topology structures as a set of general undirected graphs in a continuous time slot, denoted by $\mathcal{G}=\left(G^{1}, \ldots, G^{t}, \ldots, G^{T}\right)$. Each snapshot in slot $t$ can be represented by $G^{t}=\left(V^{t}, E^{t}\right)$, where $V^{t}$ denotes the set of nodes and $E^{t}$ denotes the set of links, respectively. Note that $V^{t}$ contains both the ground vehicle and UAVs in $t$. It is worth mentioning that the general undirected graph will be converted to a geometric graph if UAVs are considered to be homogeneous, i.e., they have the same communication range.

\section{B. Mobility Model}

To create a more realistic mobility pattern, several mobility models have been specifically designed for FANETs. Meanwhile, there also exists some mobility models that were originally introduced for MANETs and now are used for FANETs. In [36], the authors discussed most existing mobility models for FANETs, e.g., random direction (RD), random waypoint (RWP), Gauss-Markov (GM), and semi-random circular movement (SRCM) mobility models, etc. However, these models cannot capture the swarm intelligence properties of UAVs in a FANET, especially for certain types of collaboration and cooperation. Hence, selecting an appropriate mobility model from those with different characteristics is improtant to getting valuable conclusions from a realistic simulation environment. To this end, we consider the swarm mobility model, where coordinated behavior of the swarm emerges from relatively simple interactions between individuals during task execution. Similar to the formation of flocks of birds, the motions of UAVs comply with the following requirements [37]: 1) UAVs adjust autonomously their directions and plan their displacement according to their neighbors' status: move away to avoid collisions when they are too close whereas get closer to keep cohesion; 2) Each UAV can join and depart arbitrarily; and 3) To keep movements coordinated, UAVs continually tend to adjust their speed to the average speed of their neighbors.

Herein, we propose a bio-inspired swarm mobility model based on the classic Boid model [37], which can accurately describe the group motion property of UAVs in a FANET. In the original Boid model, three rules, i.e., Cohesion, Separation, Alignment rules are defined. As shown in Fig.2, to mimic the behavior of a Boid, the sensing area of a UAV can be divided into three zones, from the farthest to the nearest one: attraction zone (cohesion rule), stability zone (alignment rule), and repulsion zone (separation rule). Wherein, the distance $d_{i, j}(t)$ between $u_{i}$ and its neighbor $u_{j}$ should satisfy

$$
R_{s} \leq d_{i, j}(t) \leq R_{c} \leq R_{u}
$$

where $R_{s}$ is the safe distance, i.e., the radius of repulsion zone, $R_{c}$ denotes the reliable communication distance, i.e., the radius of stability zone, and $R_{u}$ represents the unstable distance, i.e., the radius of attraction zone.

When its neighbors enter the repulsion zone, stability zone, or attraction zone, the UAV moves away to avoid collisions, keeps following it, or moves towards it, respectively. However, the previously defined three rules cannot describe the swarm motion properity of the whole UAV team very well, because only the movements of neighbor nodes are considered. In this regard, we further introduce three novel rules, called centripetalism, consistency, and synchronization rules. All UAVs can obtain the position information of other UAVs within their sensing ranges by mantaining the networking connectivity. During the flight, each UAV $u_{i}$ has a velocity vector $\vec{v}_{i}(t)$ and its current position vector can be denoted as $\vec{p}_{i}(t)$. The detailed descriptions of six rules and their formulas expressions are shown as follows.

- Cohesion Rule: This rule depicts the feature that the motion of a single UAV $u_{i}$ is determined by its neighbor set $N B_{i}^{a}(t)$ within attraction zone to some extent, so that each UAV will move towards the center of $N B_{i}^{a}(t)$. The motion component generated by cohesion rule can be formulated as

$$
\overrightarrow{D_{i}^{1}}(t+1)=\frac{\sum_{u_{k} \in N B_{i}^{a}(t)}\left(\overrightarrow{p_{k}}(t)-\vec{p}_{i}(t)\right)}{\left|N B_{i}^{a}(t)\right|}
$$

- Alignment Rule: This rule ensures that each UAV will adapt its velocity direction to those of its neighbors.

$$
\overrightarrow{D_{i}^{2}}(t+1)=\frac{\sum_{u_{k} \in N B_{i}^{a}(t)} \overrightarrow{v_{k}}(t)}{\left|N B_{i}^{a}(t)\right|}-\overrightarrow{v_{i}}(t) .
$$

- Separation Rule: This rule ensures that when some UAVs enter $u_{i}$ 's repulsion zone, it will generate an opposite motion component against the center of its neighbor set $N B_{i}^{r}(t)$ within the repulsion zone.

$$
\overrightarrow{D_{i}^{3}}(t+1)=\frac{\sum_{u_{j} \in N B_{i}^{r}(t)}\left(\overrightarrow{p_{i}}(t)-\overrightarrow{p_{j}}(t)\right)}{\left|N B_{i}^{r}(t)\right|} .
$$

- Centripetalism Rule: If we only consider the cohesion rule, UAV swarm may be dispersed into small groups. In this case, the motion of a single UAV $u_{i}$ is also determined by the UAV swarm.

$$
\vec{D}_{i}^{4}(t+1)=\frac{\sum_{u \in N(t)}\left(\overrightarrow{p_{u}}(t)-\vec{p}_{i}(t)\right)}{|N(t)|} .
$$


- Consistency Rule: To better imitate the group movement, each UAV will adapt its velocity direction to the UAV swarm.

$$
\overrightarrow{D_{i}^{5}}(t+1)=\frac{\sum_{u \in N(t)} \overrightarrow{v_{u}}(t)}{|N(t)|}-\overrightarrow{v_{i}}(t) .
$$

- Synchronization Rule: Considering the limited vision of a single UAV, it can only move towards what it thinks is the optimal direction. If its neighbors' directions are very chaotic, it can get the average motion direction strategically, but this direction cannot delineate the synchronous direction around it very well. In this case, it should adopt a relatively conservative strategy, that is, although the average direction of motion was obtained and the direction of motion was adjusted, due to the uncertainty of this direction, it can slow down to avoid flight confusions with extra adjustment costs. Only if its neighbors have reached synchronization, it will fly at its maximum speed. We introduce a parameter $s y n_{i}$ to denote the synchronization degree of $u_{i}$ against global swarming for the velocity calculation.

$$
\operatorname{syn}_{i}=\frac{\left|\sum_{u_{k} \in N B_{i}^{a}(t)} \overrightarrow{v_{k}}(t)\right|}{\sum_{u_{k} \in N B_{i}^{a}(t)}\left|\overrightarrow{v_{k}}(t)\right|} .
$$

Note that $\operatorname{syn}_{i}$ is within the interval $[0,1]$. The higher the value, the higher the synchronization degree of the global swarm. Then, the absolute value of velocity in the next slot can be given by

$$
\left|\vec{v}_{i}(t+1)\right|=V_{\max } e^{\epsilon\left(s y n_{i}-1\right)}
$$

where $\epsilon$ denotes the adjustable coefficient.

Each of rules can produce a motion component and all of the components determine the motion. Note that the weight of each component is determined by different application scenarios. Based on the above discussion, the motion direction in the next slot can be expressed as

$$
\begin{aligned}
\vec{D}_{i}(t+1) & =\frac{\omega_{0} \vec{v}_{i}(t)}{\left|\vec{v}_{i}(t)\right|}+\frac{\omega_{1} \overrightarrow{D_{i}^{1}}(t)}{\left|\overrightarrow{D_{i}^{1}}(t)\right|}+\frac{\omega_{2} \overrightarrow{D_{i}^{2}}(t)}{\mid \overrightarrow{D_{i}^{2}(t) \mid}} \\
& +\frac{\omega_{3} \vec{D}_{i}^{3}(t)}{\left|\overrightarrow{D_{i}^{3}}(t)\right|}+\frac{\omega_{4} \overrightarrow{D_{i}^{4}}(t)}{\left|\overrightarrow{D_{i}^{4}}(t)\right|}+\frac{\omega_{5} \overrightarrow{D_{i}^{5}}(t)}{\left|\overrightarrow{D_{i}^{5}}(t)\right|}
\end{aligned}
$$

where the first item $\overrightarrow{v_{i}}(t) /\left|\overrightarrow{v_{i}}(t)\right|$ denotes the inertial movement component, since the current motion status has an impact on the motion for next moment. $\omega_{0}, \omega_{1}, \omega_{2}, \omega_{3}, \omega_{4}$, and $\omega_{5}$ are different weighting factors with the constraint of $\omega_{0}+\omega_{1}+\omega_{2}+\omega_{3}+\omega_{4}+\omega_{5}=1$. Herein, we set equal weights.

Besides, the position vector in the next slot can be given by

$$
\vec{p}_{i}(t+1)=\vec{p}_{i}(t)+\vec{D}_{i}(t)\left|\vec{v}_{i}(t)\right| \delta .
$$

\section{Energy Model}

Similar to the power model designed for multi-rotor UAVs [38], we consider three types of power consumption in the flight duration, i.e., the induced power $P^{i n}$, the profile power $P^{p r}$, and the parasite power $P^{p a}$. Given the gravity $m g$ of a UAV $u_{i}$, its total thrust $\tau_{i}(t)$ in $t$ can be represented by $\tau_{i}(t)=$ $\sqrt{\left(m g-\left(c_{5}\left(V_{i}^{\text {ver }}(t) \cos \theta\right)^{2}+c_{6} \tau_{i}(t)\right)\right)^{2}+\left(c_{4}\left(V_{i}^{\text {hor }}(t)\right)^{2}\right)^{2}}$. Note that the vertical speed and horizontal speed can be calculated by $\overrightarrow{v_{i}}(t) \cos \phi_{i}(t)$ and $\overrightarrow{v_{i}}(t) \sin \phi_{i}(t)$, where $\phi_{i}(t)$ denotes the angle between the movement direction and z-axis and $\theta$ denotes the angle of attack. Then, the induced power $P_{i}^{i n}$ which generates thrust by propelling air downward can be formulated as

$$
P_{i}^{i n}(t)=k_{1} \tau_{i}(t)\left[\frac{V_{i}^{\text {ver }}(t)}{2}+\sqrt{\frac{V_{i}^{\text {ver }}(t)}{2}}+\frac{\tau_{i}(t)}{k_{2}^{2}}\right] .
$$

By means of the rotating propeller blades, the generated profile power $P_{i}^{p r}$ can overcome the encountered rotational drag, which can be represented by

$$
P_{i}^{p r}(t)=c_{2} \tau_{i}(t)^{3 / 2}+c_{3}\left(V_{i}^{h o r}(t) \cos \theta\right)^{2} \tau_{i}(t)^{1 / 2},
$$

When there exists a relative translational motion between the UAV and wind, the parasite power $P_{i}^{p a}$ is utilized to resist body drag, which can be expressed by

$$
P_{i}^{p a}(t)=c_{4}\left(V_{i}^{h o r}(t)\right)^{3},
$$

where the notations $k_{1}, k_{2}, c_{2}, c_{3}, c_{4}, c_{5}$, and $c_{6}$ in Eq. (15), Eq. (16) and Eq. (17) are constant parameters related to the physical characteristics, which can be refered to [38] for more details.

Then, the flight power in slot $t$ can be approximated by:

$$
P_{i}^{f}(t)=P_{i}^{i n}(t)+P_{i}^{p r}(t)+P_{i}^{p a}(t) .
$$

Without loss of generality, we neglet the transmit power part in the power model, because transmit power is scales of magnitude lower than the flight power [38]. Here, we focus on the current energy consumption before the next charging, termed as $E_{i}^{k}(t)$. Given the flight duration from the last time the battery has been charged (i.e., $T_{i}^{k-1}$ ) to the current slot $t$, the total energy consumed by the $u_{i}$ during its $k$-th flight duration is given by:

$$
E_{i}^{k}(t)=\sum_{j=T_{i}^{k-1}}^{t} P_{i}^{f}(j) \delta .
$$

Note that $E_{i}^{k}(t)$ is reset when a UAV $u_{i}$ leaves for recharing. If the residual energy of $u_{i}$ is less than a minimum allowabe value, it will enter tracking mode and then adjust its velocity direction to return to the top of the vehicle for recharing or battery swapping. Similar to [39], we consider that each UAV chooses to swap battery and thus the $k$-th charging duration of the UAV $u_{i}$, i.e., $T_{i}^{c h}(k)$, can be measured by the tracking time, which can be transformed to a chase problem whose solution can be obtained by solving some simultaneous equations at discrete moments. The specific calculation process is omitted due to the limited space and its simplicity. 


\section{Problem Definition}

In this section, we first define the adaptive VBN maintenance problem based on the presented models in the previous section, and then transform this problem to a dynamic minimal $d$-CDS problem in graph theory to minimize the routing overhead while ensuring that the maintenance cost to deal with topology changes is reduced as much as possible.

As discussed in Section I, due to some unique features of FANETs, it is challenging to establish stable multi-hop links relaying alert messages through a series of intermediate nodes while incorporating a maintenance strategy in the case of frequently intermittent connectivity. Generally, building a VBN is widely used for simplifying the routing problem to reduce most routing and forwarding operations into partial nodes. Different from most existing works that are dedicated to low dynamic networks, the VBP construction in FANETs should adapt to a network comprising nodes with high mobility. Through mapping, nodes in VBN correspond to dominators and nodes outside VBN correspond to dominatees, which indicates that the VBN can be abstracted as a CDS. Given a set of general undirected graph $\mathcal{G}=\left(G^{1}, \ldots, G^{t}, \ldots, G^{T}\right)$ abstracted from time-varying networking topologies, we hope to adaptively maintain the minimal $d$-CDS for each snapshot. The minimal $d$-CDS can be defined as follows.

Definition 1 [14]: For a given graph $G=(V, E)$, a $d$-DS denotes a vertex set $\mathcal{D}$ such that any vextex in $V$ is either in set $\mathcal{D}$ or connected to a node in set $\mathcal{D}$ within $d$-hop distance ( $d$-hop dominated by $\mathcal{D})$. A $d$-CDS $\widetilde{\mathcal{D}}$ induced from $\mathcal{D}$ adds the constraint that any two vertices in $\widetilde{\mathcal{D}}$ must be connected. The vertices are called dominators and dominatees in $\widetilde{\mathcal{D}}$ and $V \backslash \widetilde{\mathcal{D}}$, respectively. The minimal $d$-CDS represents the $d$-CDS with minimal size.

Theorem 1 [22], [31]: Both minimal $d$-DS and minimal $d$-CDS problems are NP-complete problems.

Proof: In [31], the authors proved that the minimal $d$-DS problem can be reduced from a NP-complete Vertex Cover problem, while the authors in [22] proved that the minimal $d$-CDS problem can be reduced from a NP-complete 3-SAT problem in polynomial time, which proves Theorem 1.

According to Theorem 1, it is natural to resort to designing approximation algorithms for minimal $d$-CDS problem due to its NP-complete nature. Generally, many existing works adopt a three-step algorithm as follows: 1) Construct a minimal d-hop independent set ( $d$-IS) since a minimal $d$-IS is equivalent to a minimal $d$-DS for a graph [15]; 2) Connect this minimal $d$-IS into a $d$-CDS; and 3) Minimize the size of the $d$-CDS by vertex delection and connectivity checking iteratively. However, when topology changes, most existing works reconstruct the minimal $d$-CDS for current slot from scratch, which is not desirable in dynamic environments since the maintenance cost is very high for each update. Generally, the maintenance cost is determined by the volume of broadcast messages during topology update phase. Since all nodes have the similar physical characteristics, the maintenance cost of each node can be represented by a constant, i.e., a certain communication overhead, and thus the average maintenance cost can be simplified as the average number of updated nodes, which can be expressed by

$$
A C=\frac{1}{T} \sum_{t=1}^{T} \sum_{i=1}^{N(t)} C_{i}(t),
$$

where

$$
C_{i}(t)= \begin{cases}1, & \text { if } u_{i} \text { will be updated in } t \\ 0, & \text { otherwise. }\end{cases}
$$

In order to approximately find a minimal $d$-CDS without causing too much maintenance cost in response to a highly dynamic environment, we should ensure that the VBN maintenance process triggered by vertex/edge insertion/deletion can involve as few vertice as possible, which indicates that the solution in $t$ should be based on that in $t-1$ and the topology changes $T C_{t-1}^{t}$ from $t-1$ to $t$ rather than selecting from scratch. Thus, the definition of adaptive topology maintenance problem, can be transformed to a dynamic minimal $d$-CDS problem, is as follows.

Definition 2: Given a set of general undirected graphs $\mathcal{G}=\left(G^{1}, \ldots, G^{t}, \ldots, G^{T}\right)$, the dynamic minimal $d$-CDS problem is to find a $d$-CDS $\widetilde{\mathcal{D}}_{t}$ with minimal size in slot $t$, while minimizing the average maintenance cost $A C$. Meanwhile, $\widetilde{\mathcal{D}_{t}}$ should be derived adaptively, according to only $\widetilde{\mathcal{D}}_{t-1}$ and $T C_{t-1}^{t}$.

The main difficulty of solving the dynamic minimal $d$-CDS problem of FANETs in emergency scenarios lies in the fact that we should strike a trade-off among the routing overhead, response time, and maintenance costs per topology update. Hereinafter, we will investigate how to balance these three performances by designing an adaptive solotion framework.

\section{AdAPtive Solution}

Following the classic three-step algorithm structure to solve the static minimal $d$-CDS problem, we first propose an approximation algorithm for maintaining a minimal $d$-DS. Then, we insert additional vertices to connect the obtained $d$-DS as a $d$-CDS to repair the connectivity. Finally, we show how to adaptively restore the minimality of the CDS based on the top-tree data structure.

\section{A. $\mathcal{O}(\log (N))$-Approximation to Minimal d-DS}

We first introduce some useful preliminaries, which facilitates the understanding of proposed algorithms. For the topology $G^{t}$ in time slot $t$, for any $v_{i} \in V(t)$, let $N B_{i}^{d}(t)$ denote the set of its $d$-hop neighbors, i.e., within $d$-hop transmission distance. Here, we want to maintain a solution $S(t)$ containing some dominating pairs $\left(v_{i}, \operatorname{Dom}\left(v_{i}\right)\right)$, where the set $\operatorname{Dom}\left(v_{i}\right) \in N B_{i}^{d}(t)$ denote those vertices that are $d$ hop dominated by $v_{i}$ and their cardinality is $\left|\operatorname{Dom}\left(v_{i}\right)\right|$. We set that a vertex can appear as the dominant vertex in many pairs whilst each vertex is $d$-hop dominated by exactly one dominator $v_{i}$, i.e., belongs to exactly one $\operatorname{Dom}\left(v_{i}\right)$. Hence, the collection of dominant vertices from all dominating pairs can denote the solution for the minimal $d$-DS problem, which is valid since each vertex is $d$-hop dominated, i.e., each vertex is exactly in one $\operatorname{Dom}\left(v_{i}\right)$. 
In [26], Gupta et al. designed a smart algorithm for solving the dynamic SC problem. As discussed before, minimal $d$-DS is equivalent to $\mathrm{SC}$ in static setting. In dynamic setting, the dynamic SC problem focuses on the dynamics of the universe $S$ covered by an unchanged family of sets $F$. But the general dynamic $d$-DS problem focuses on the dynamics of a set of dominators (in analogy with $F$ ) while the set of vertices is unchanged (in analogy with $S$ ). Based on [26], Hjuler et al. investigated how to tackle the general dynamic 1-DS problem with unchanged vertex set, i.e., $S$, by only considering the dynamics of edges [33]. Inspired by these two works, we want to deal with a more general case, i.e., both $F$ and $S$ are dynamically changing for $d \geq 1$.

The main idea of our scheme to solve the dynamic $d$-DS relies partly on the hierarchy strategy [26]: the dominating pairs are assigned to different layers based on their cardinality. More specifically, the capacity of layer $l$ falls within the range $R^{l}=\left[2^{l-10}, 2^{l}\right]$ and a dominating pair $\left(v_{i}, \operatorname{Dom}\left(v_{i}\right)\right)$ is dominated at layer $l$ if $\left|\operatorname{Dom}\left(v_{i}\right)\right| \in R^{l}$. We denote $V_{l}(t)$ as the set of all vertices belonging to layer $l$. In this case, an assignment of layers is reseonable for $\left(v_{i}, \operatorname{Dom}\left(v_{i}\right)\right)$ if the constraint $\left|\operatorname{Dom}\left(v_{i}\right)\right| \in R^{l}$ is always satisfied. Thus, the stability of a solution can be defined as in the following definition.

Definition 3: In slot $t$, a stable solution $S(t)$ satisfies that there exists no vertex $v_{i}$ and layer $l$ such that $\left|V_{l}(t) \bigcap N B_{i}^{s}(t)\right|>2^{l}$, which means that it is impossible to introduce a new vertex $v_{j} \in V_{l}(t)$ as a dominator to dominate some vertices in $V_{l}(t)$ such that the newly formed dominating pair could be assigned to the layer whose level is strictly greater that $l$.

Based on the stability definition, we will dynamically maintain a stable solution. To prevent too many layer changes, we allow overlapping ranges $R^{l}$ to give some slack to our algorithm. Initially, we consider vertex degree, average estimated connection duration, and residual energy as three metrics to select dominators. In particular, let $C D_{i, j}(t)$ denote the estimated connection duration between $v_{i}$ and its $d$-hop neighbor $v_{j} \in N B_{i}^{d}(t)$, which can be deduced as [16]

$$
C D_{i, j}(t)=\min _{p, q \in P(i, j)(t)} \sqrt{\left(R_{c}^{2}-L_{p, q}^{2}(t)\right) /\left(\overrightarrow{v_{p}}(t)-\overrightarrow{v_{q}}(t)\right)^{2}}
$$

where $P(i, j)(t)$ denotes the vertex set along the path from $v_{i}$ to $v_{j}, \overrightarrow{L_{p, q}^{2}}(t)$ is the relative distance between $v_{p}$ and $v_{q}$, and the term $\overrightarrow{v_{p}}(t)-\overrightarrow{v_{q}}(t)$ denotes their relative velocity. E. q. (22) indicates that the connection duration between two vertices within $d$-hop is determined by the one-hop link with shortest duration. Then, the average estimated connection duration can be represented by

$$
A C D_{i}(t)=\frac{\sum_{v_{j} \in N B_{i}^{d}(t)} C D_{i, j}(t)}{\left|N B_{i}^{d}(t)\right|} .
$$

Herein, we use a variable $I_{i}(t)$ to measure its dominant level, which can be denoted as

$$
I_{i}(t)=\varpi_{1} \overline{N B_{i}^{d}}(t)+\varpi_{2} \overline{R E_{i}}(t)+\varpi_{3} \overline{A C D_{i}}(t),
$$

where $\overline{\operatorname{deg}_{i}}(t)$ denotes the normalized $d$-hop neighbor number, i.e., $\overline{N B_{i}^{d}}(t)=N B_{i}^{d}(t) / N(t), \overline{R E_{i}}(t)$ denotes the percentage of residual energy, and $\overline{A C D_{i}}(t)$ denotes the normalized $A C D_{i}(t)$, which can be calculated by the ratio of $A C D_{i}(t)$ to $\min _{p, q \in P(i, j)(t)} \sqrt{R_{c}^{2} /\left(\overrightarrow{v_{p}}(t)-\overrightarrow{v_{q}}(t)\right)^{2}} \cdot \varpi_{1}, \varpi_{2}$, and $\varpi_{3}$ are different weighting factors with the constraint of $\varpi_{1}+\varpi_{2}+$ $\varpi_{3}=1$. Herein, we set equal weights. Note that we can exploit the more complex analytic hierarchy process (AHP) and entropy weight generation method to construct $I_{i}(t)$. Since this part is not our main focus, we select the widely used normalization method.

Based on the above discussion, the dominators are elected according to $I_{i}(t)$ in a descending order, and then the dominating pairs are formed one by one. This process terminates when all vertices are dominated. When the topology changes, we want to restore the stability at the end of every update. Based on this idea, the adaptive minimal $d$-DS (AMDD) algorithm is presented in Alg. 1. Note that for edge addition/deletion, the two endpoints of this edge should exist in both $t-1$ and $t$. The procedure $\operatorname{Stabilize}\left(v_{i}, l\right)$ is summarized in Alg. 2. All the vertices are dominated in $S(t)$ for each $t$ since Stabilize does not make any node undominated, which proves the correctness of Alg. 1. It is worth mentioning that the dominant level can still be ensured in another way. Firstly, the stability condition will ensure that $\mathcal{D}_{t}$ always includes those dominators with a large amount of $d$-hop neighbors. Besides, those newly arrived vertices (fully charged) will be included. Finally, our mobility model can control the mutual distance and thus the connection duaration is also ensured. Next, we will analyze its approximation ratio and time complexity for each update.

Theorem 2: Alg. 1 has an approximation ratio of $\mathcal{O}(\log (N))$, where $N$ denotes the maximal number of vertices in initial stage.

Proof: For each dominating pair $\left(v_{i}, \operatorname{Dom}\left(v_{i}\right)\right)$, its cardinality satisfies the condition $\left|\operatorname{Dom}\left(v_{i}\right)\right| \leq N$. In this case, $\log (N)$ layers are enough to contain all pairs. Thus, the cost for maintaining a stable solution is at most $\mathcal{O}(\log (N)) \cdot \mathrm{Opt}$, where Opt is the optimal cost.

For time complexity, we measure the amount of time taken to update the solution $S_{t-1}$ to $S_{t}$. To better depict this property, we will apply the amortized analysis by firstly giving the upper bound for the number of vertices that change level and then showing how to implement a level change with $\mathcal{O}(\Delta)$ costs, where $\Delta$ denotes the maximal degree of a vertex over the sequence of updates.

\footnotetext{
Algorithm 1 Adaptive Minimal $d$-DS (AMDD) Algorithm

1: Proceed to the next slot $t$

2: Let $T C_{t-1}^{t}$ denotes the topology changes from $t-1$ to $t$. An element $\rho \in T C_{t-1}^{t}$ can be divided into four categories: vertex addition/ deletion and edge addition/deletion.

3: while $T C_{t-1}^{t} \neq \emptyset$ do

4: $\quad$ Randomly choose an element $\rho$ from $T C_{t-1}^{t}$.

5: $\quad$ if $\rho$ denotes the vertex addition of $v_{i}$ then

6: $\quad$ Add the pair $\left(v_{i}, \operatorname{Dom}\left(v_{i}\right)=v_{i}\right)$ to $S(t)$ with layer 1. Insert edges between $v_{i}$ and its neighbors.
} 


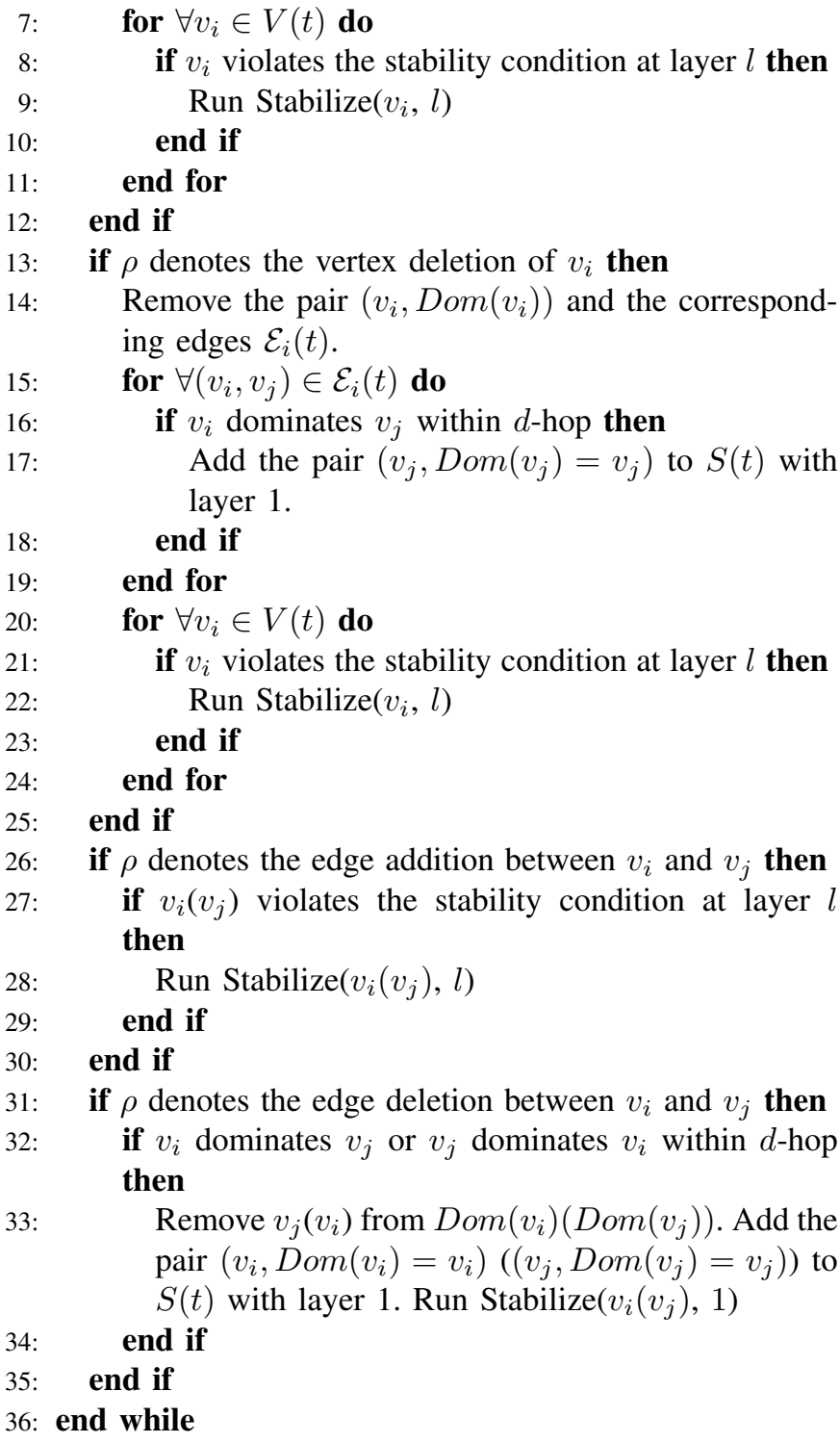

Lemma 1: After $k$ updates in $t$, the totoal amount of vertices that have changed layer levels is at most $\mathcal{O}(k d \Delta \log (N)+$ $N \log (N))$.

Proof: Herein, we adopt the token scheme in [26], where every vertex is initially assigned $2 \log (N)$ tokens and pays one token for each layer level change. Note that the "token transfer" here is to bound the number of vertices that change level. We use the token scheme to facilitate the amortized analysis by explaining how to implement a level change so that it costs $\mathcal{O}(\Delta)$. Besides, an undominated vertex will be assigned $2 \log (N)$ new tokens. For each edge deletion, at most $d$ vertex lose its dominator. Considering that each vertex is involved in at most $\Delta$ edges, at most $d \Delta$ vertices transform to the undominated status for each vertex deletion. Thus, after $k$ updates, the total amount of consumed tokens is bounded by $\mathcal{O}(k d \Delta \log (N)+N \log (N))$. To further prove this point, the following condition should be satisfied, which indicates that the amount of tokens of each vertex is always positive.

Proposition 1: The total amount of tokens of each vertex at layer $l$ is more than $2 \log (N-1)$.

Proof: As discussed above, one token is expended when

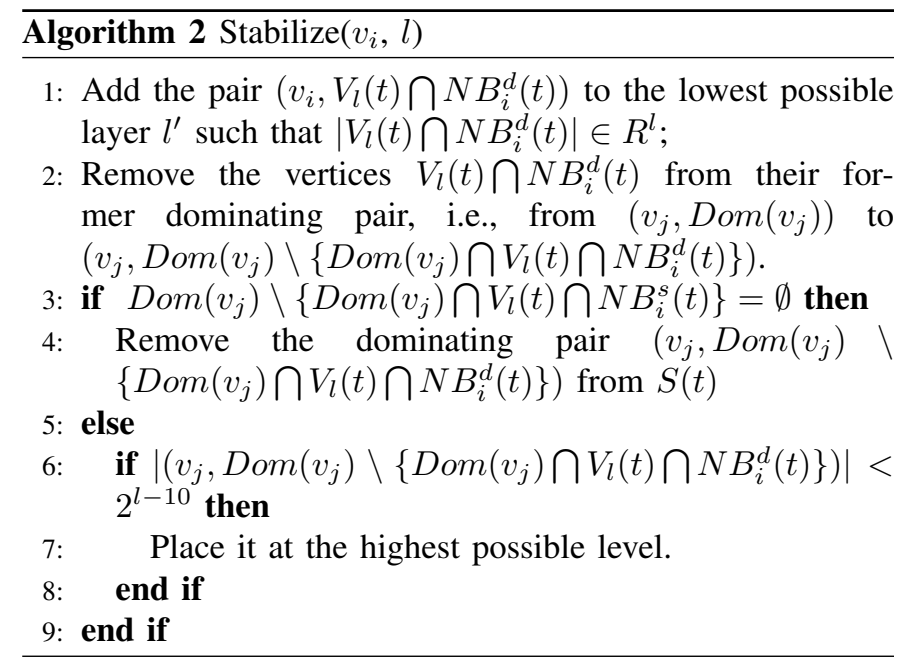

a vertex moves to a higher layer. Besides, it also has an "emergency fund" to "atone" for deserting its siblings in its former dominating pair. It will save one token and then donate it to the emergency fund, which will come in handy when its remaining siblings move to another layer. When the pair $\left(v_{i}, \operatorname{Dom}\left(v_{i}\right)\right)$ has a level change $\Delta l$, i.e., moving from layer $l$ to $l-\Delta l$, several vertices must have left from $\operatorname{Dom}\left(v_{i}\right)$ and their paid tokens can be used for the operation. Hence, each vertex in $\operatorname{Dom}\left(v_{i}\right)$ needs $2 \Delta l+1$ tokens. We can further get $\left|\operatorname{Dom}\left(v_{i}\right)\right|<2^{l-j}$ due to the condition that $\left(v_{i}, \operatorname{Dom}\left(v_{i}\right)\right)$ has been moved to $l-\Delta l$ layer. Moreover, the cardinality of $\operatorname{Dom}_{b}\left(v_{i}\right)$ that was created before the change satisfies $\left|\operatorname{Dom}_{b}\left(v_{i}\right)\right|>2^{l-1}$, which indicates that the amount of usable tokens is larger than $2^{l-1}-2^{l-\Delta l}$. Next, we need to further prove that $2^{l-1}-2^{l-\Delta l} \geq(2 \Delta l+1)\left|\operatorname{Dom}\left(v_{i}\right)\right|$. Since $\left|\operatorname{Dom}\left(v_{i}\right)\right|<2^{l-\Delta l}$, if we prove that $2^{l-1}-2^{l-\Delta l} \geq$ $(2 \Delta l+1) 2^{l-\Delta l}$, i.e., $2^{\Delta l-1}-1 \geq(2 \Delta l+1)$, the above inequality holds. Considering that the pair was moved to $l-\Delta l$ layer, both the inequalities $\left|\operatorname{Dom}\left(v_{i}\right)\right|>2^{l-\Delta l-1}$ and $\left|\operatorname{Dom}\left(v_{i}\right)\right|<2^{l-10}$ holds and then we can get $\Delta l>9$. Pluging in this condition and then we can get $2^{\Delta l-1}-1 \geq$ $(2 \Delta l+1)$, which concludes the proof.

Lemma 2: Alg. 1 can maintain a stable solution in $\mathcal{O}\left(d \Delta^{2} \log (N)\right)$ amortized time per update.

Proof: Alg. 1 maintains the set $V_{l}(t) \bigcap N B_{i}^{d}(t)$ and its cardinality for all $v_{i}$ and layer $l$. When a vertex moves to another layer, all its neighbor will be informed, which costs $\mathcal{O}(\Delta)$. For any types of topology changes involving $v_{i}$, Alg. 1 updates the set $V_{l}(t) \bigcap N B_{i}^{d}(t)$. When invoking Alg. 2, we need to maintain a vertex list containing those vertices violating the stability. Alg. 2 deals with this list vertex by vertex to restore the stability in constant time. Since we need to pay $\mathcal{O}(\Delta)$ per layer change and the amortized changes are bounded by $\mathcal{O}(d \Delta \log (N))$, the amortized time complexity of each update is $\mathcal{O}\left(d \Delta^{2} \log (N)\right)$.

\section{B. Adaptive Solution to Minimal d-CDS}

Based on the $d$-DS $\mathcal{D}_{t}$ obtained by Alg. 1, we have to add a set of vertices $\mathcal{C}_{t}$ to ensure that $\widetilde{\mathcal{D}}_{t}=\mathcal{C}_{t} \cup \mathcal{D}_{t}$ is connected and minimal. Overall, $\mathcal{C}_{t}$ has to be updated for two reasons: 
- For cases of edge/vertex deletion caused by topology changes or vertex removal caused by Alg. 1, some vertices need to be added to $\mathcal{C}_{t}$ to rapair the connectivity.

- For cases of edge/vertex insertion caused by topology changes, Alg. 1, or connectivity restoration, some useless vertices of $\mathcal{C}_{t}$ need to be further removed to maintain the minimality.

In the first step, we have to identify which vertices need to be added to $\mathcal{C}_{t}$ to repair the connectivity. We first deal with the case of edge deletion. To detect the connectivity, we maintain a MST of each snapshot $G^{t}$ in which each edge in $\widetilde{\mathcal{D}}_{t}$ is assigned with a weight 1 and other edges are assigned with a weight $w$. If $\widetilde{\mathcal{D}}_{t}$ is connected, the MST extend a tree on $\widetilde{\mathcal{D}}_{t}$. Otherwise, the MST adds an internal vertex in $V(t) \backslash \widetilde{\mathcal{D}}_{t}$ to $\mathcal{C}_{t}$ to repair the connectivity. It is worth mentioning that the edge weights are updated in cases of vertex/edge insertion/deletion. The weighted MST has the following properties: 1) If $\widetilde{\mathcal{D}}_{t}$ is a $d$-CDS, the sum weights of this MST is $\left(\left|\widetilde{\mathcal{D}}_{t}\right|-1\right)+w$. $\left|V(t) \backslash \widetilde{\mathcal{D}}_{t}\right|$ by using $\left|\widetilde{\mathcal{D}}_{t}-1\right|$ edges of weight 1 to construct a spanning tree on $\widetilde{\mathcal{D}}_{t}$ and $\left|V(t) \backslash \widetilde{\mathcal{D}}_{t}\right|$ edges of weight $w$ to induce the $G^{t} ; 2$ ) Otherwise, the sum weights of the MST will be larger than $\left(\left|\widetilde{\mathcal{D}}_{t}\right|-1\right)+w \cdot\left|V(t) \backslash \widetilde{\mathcal{D}}_{t}\right|$.

For any two connected components $C_{1}$ and $C_{2}$ in $\widetilde{\mathcal{D}}_{t}$, the insertion of those vertices along the shortest path between $v \in C_{1}$ and $u \in C_{2}$ is the least costly way to repair the connectivity, i.e., involving the minimum number of vertices. It is worth mentioning that the length of this path is at most $2 d+1$. Otherwise, there must be a vertex not $d$-hop dominated by any vertex in $\mathcal{D}_{t}$. After removing an edge, the connectivity failure of $\widetilde{\mathcal{D}}_{t}$ may occur. In this case, the MST will involve at most $2 d$ internal vertices not in $\mathcal{D}_{t}$, which should be added to $\mathcal{C}_{t}$ to ensure the connectivity. Since edge insertion has no influence on the connectivity, we then deal with vertex insertion/deletion.

If a vertex is deleted from $\widetilde{\mathcal{C}_{t}}$ caused by topology changes and $\widetilde{\mathcal{D}}_{t}$ becomes disconnected, the MST involves at most $2 d \Delta$ internal vertices to $\mathcal{C}_{t}$ to ensure the connectivity, since the deletion of each vertex in $\widetilde{\mathcal{C}}_{t}$ disconnects at most $\Delta$ components and at most $2 d$ vertices are needed to connect two components.

For other types of changes, Alg. 1 will first reconstruct the $d$-DS. If a vertex $v$ is added to or deleted from $\mathcal{D}_{t}$ by Alg. 1, we should be more careful: updating the MST may add several vertices to $\mathcal{C}_{t}$ (at most $\Delta$ ). If $\widetilde{\mathcal{D}}_{t}$ get disconnected after the deletion of $v, v$ is added to $\mathcal{C}_{t}$. Otherwise, nothing needs to be done. If the addition of $v$ disconnects $\widetilde{\mathcal{D}}_{t}$, the MST includes at most $2 d$ internal vertices to $\mathcal{C}_{t}$ to restore the connectivity. Then, we utilize the fully-dynamic MST algorithm with time complexity $\mathcal{O}\left(\log ^{4}(N)\right)$ in [40] to maintain the weighted MST on each snapshot. Thus, the time complexity can be bounded by $\widetilde{\mathcal{O}}(\triangle)$, because the weights of edges connected to the added internal vertices are also updated, .

The second step is to restore the minimality of $\mathcal{C}_{t}$, because some existing vertices in $\mathcal{C}_{t}$ may be invalid for the connectivity of $\widetilde{\mathcal{D}}_{t}$. In graph theory, an articulation point denotes the vertex whose removal disconnects the graph and thus the number of connected components is increased. To restore the minimality of $\mathcal{C}_{t}$, all vertices in $\mathcal{C}_{t}$ are required to be articulation points in the graph induced by $\widetilde{\mathcal{D}}_{t}$.

Note that some new vertices are introduced to $\mathcal{C}_{t}$ or $\mathcal{D}_{t}$ to restore the connectivity in the previous step. It may happen that some old vertices in $\mathcal{C}_{t}$ are not articulation points anymore. In this case, the most obvious approach is to query all the vertices $v \in \mathcal{C}_{t}$ one-by-one to detect whether or not $\widetilde{\mathcal{D}}_{t} \backslash v$ is still connected. However, this approach needs $\widetilde{\mathcal{O}}(N)$ time complexity to traverse all vertices, which can be further reduced by narrowing down the search. To this end, we will maintain another unweighted spanning forest $\mathcal{F}_{t}$. As the name implies, a spanning forest is composed of more than one spanning trees.

Lemma 3: After the insertion of an edge $(v, w)$, those vertices in $\mathcal{C}_{t}$ that are not articulation points anymore belong to the tree path from $v$ to $w$. After removing these vertices along tree path, other vertices become articulation points again.

Proof: Let $G_{b}^{t}$ and $G_{a}^{t}$ denote the graphs before and after the insertion of $(v, w)$, respectively. Besides, $u$ is an articulation point in $\widetilde{\mathcal{D}}_{t}^{b}$ induced by $G_{b}^{t}$ but not in $\widetilde{\mathcal{D}}_{t}^{a}$ induced by $G_{a}^{t}$.

Assuming that $u$ is not on the tree path from $v$ to $w, v$ and $w$ are connected in $G_{b}^{t} \backslash u$. Besides, $v$ is not connected to some vertices $x$ in $G_{b}^{t}$, because $u$ is an articulation point in $\widetilde{\mathcal{D}}_{t}^{b}$. For such situation, the insertion of $(v, w)$ has no impact on the disconnection between $v$ and $x$, which indicates that $u$ is still an articulation point. Hence, we can deduce that all articulation points that could be deleted must belong to the cycle $v \ldots w \ldots v$. Considering that those vertices are not articulation points in $\widetilde{\mathcal{D}}_{t}^{a}, G_{b}^{t}$ is divided into only two components containing $v$ and $w$ separately. Thus, the above circle $v \ldots w \ldots v$ is the only one involving $v$ and $w$. If we delete any vertex from this circle, the articulation points in $G_{b}^{t}$ are still articulations points in $G_{a}^{t}$, because they disconnect $v$ and $w$, which contradicts that $u$ is not a articulation point in $G_{a}^{t}$.

When a vertex is added to $\widetilde{\mathcal{D}}_{t}$, all edges adjacent to its neighbors are also added one-by-one. Besides, the edge deletion from $\widetilde{\mathcal{D}}_{t}$ also affects the process of articulation point transitions. Hence, we could focus on the edge insertion/deletion. The Lemma 3 paves the new way to find those invalid vertices in $\mathcal{C}_{t}$ : finding those vertices that are no longer articulation points after inserting $(v, w)$. To this end, each vertex $v \in \mathcal{C}_{t}$ is assigned a value $\nu(v)$ which denotes the number of connected component in $\widetilde{\mathcal{D}}_{t} \backslash v$ and each $v \notin \mathcal{C}_{t}$ is assigned a value $\nu(v)$ which denotes the number of connected components plus $N$. When $(v, w)$ is inserted, if the value of one vertex $u$ on the tree path from $v$ to $w$ satisfies $\nu(u)=1, u$ is no longer an articulation point and can be directly removed from $\mathcal{C}_{t}$. Then, the removal of $u$ results in all the other vertices in $\mathcal{C}_{t}$ being articulation points again. When one vertex becomes part of $\mathcal{C}_{t}$, its value has to be decreased by $N$ for consistency. We now show how to maintain this value dynamically. To achieve this, we utilize the biconnectivity data structure called toptree on $\widetilde{\mathcal{D}}_{t}$, which is efficient to deal with the dynamic graph problems such as connectivity, minimum spanning forest, 2edge connectivity and biconnectivity [40].

The main idea is to maintain a spanning forest $\mathcal{F}_{t}$ on $\mathcal{G}^{t}$ and assign a level $l(e)$ to each non-tree edge $e \in V(t)$ but no level to the tree edges. Although we deal with the $\widetilde{\mathcal{D}}_{t}$, we focus on 


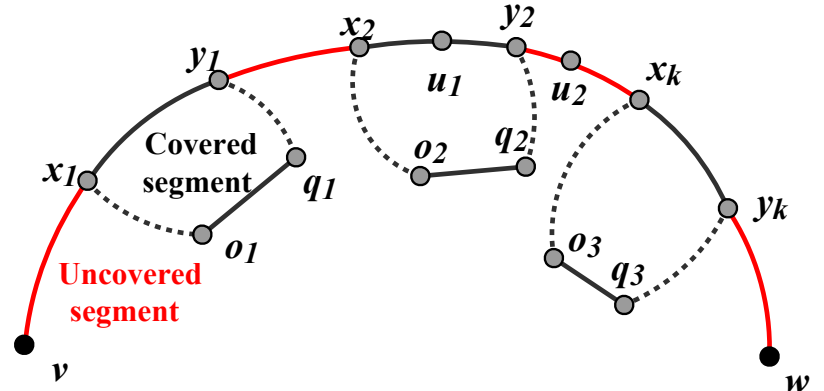

Fig. 3: Covered and uncovered segments along a tree path.

the $\mathcal{G}^{t}$ to avoid cumbersome notation, which can be achieved by removing from $\mathcal{G}^{t}$ all adjacent edges to the vertices not in $\widetilde{\mathcal{D}}_{t}$ and then the number of vertices remains $N(t)$. Let $G_{i}^{t}$ be the subgraph consisting of $\mathcal{F}_{t}$ and induced by all edges of level at least $i$, which can be maintained according to the following invariant:

Invariant 1 [40]: In a 2-edge connected component of $G_{i}^{t}$, its maximal number of vertices is $\lfloor N / i\rfloor$. Hence, the maximal relevant level $L$ satisfies $L=\left\lfloor\log _{2}(N)\right\rfloor$.

Hence, $F_{t} \subseteq G_{L}^{t} \subseteq \ldots \subseteq G_{1}^{t} \subseteq G_{0}^{t}=G^{t}$. Note that two vertices $v$ and $w$ are 2-edge connected if and only if they are connected in $\mathcal{F}_{t}$ and all edges along the tree path $v \ldots w$ are covered. A 2-edge connected component means that any two vertices of this component are 2-edge connected. Initially, all non-tree edges have level 0 , and hence the invariant 1 is satisfied. Then, it is legal to increase the level of a non-tree edge $e$ to $j$ if this does not violate invariant 1. For each tree edge $e \in F_{t}$, we attribute it a cover level $c_{l}(e)$, which denotes the maximal level from all non-tree edges covering $e$. If $e$ is a bridge whose removal disconnects a component, i.e., not covered by any non-tree edge, $c_{l}(e)=-1$.

Based on the above discussion, we should focus on the updated cover level of each tree edge and then detect which vertices are affected. As discussed before, all types of topology changes can be simplified as edge deletion/insertion. For the removal of an edge $(v, w)$, the 2-edge connectivity along the tree path $v \ldots w$ may be affected. The authors in [40] designed an algorithm to update the cover information after removing an edge, whose main idea is summarized as follows: If $(v, w)$ is a bridge, this algorithm simply remove it. If $(v, w)$ is a tree-edge but not a bridge, this algorithm replace it with another non-tree edge covering $(v, w)$ with same level in $F_{t}$, and then delete $(v, w)$. To deal with the deletion of the non-tree edge $(v, w)$, this algorithm first assign the -1 value to the cover levels of the edges in $v \ldots w$. Next, it iterates over non-tree edges $(x, y)$ covering the edges along $v \ldots w$ and update their cover levels. This step is invoked for level from $l(v, w)$ to 0 , which is correct and runs in $\widetilde{\mathcal{O}}\left(\log ^{4}(N(t))\right)$ amortized time. Interested readers can refer to [40] for more details about this algorithm and how to achieve it using the top-tree data structure.

Turning back to our problem, we care about the changes of a value $\nu(v)$ which denotes the number of connected component in $\widetilde{\mathcal{D}}_{t} \backslash v$. Considering we can obtain the covel level of each edge along the tree path $v \ldots w$, we should associate this information with value $\nu$. As shown in Fig. 3, the black segments are covered by non-tree edges while the red segments are uncovered. For the vertex $u_{1}$ in a covered segment, the connected component of $\widetilde{\mathcal{D}}_{t} \backslash u_{1}$ remain unchanged and thus $\nu\left(u_{1}\right)=1$, which means that $u_{1}$ is no longer an articulation point and can be directly removed from $\mathcal{C}_{t}$. For the vertex $u_{2}$ in an uncovered segment, $v$ and $w$ get disconnected in $\widetilde{\mathcal{D}}_{t} \backslash u_{2}$ and thus $u_{2}$ should be reserved. For the vertex in the intersection between a covered segment and an uncovered segment, e.g., $x_{1}$, its removal also disconnects $v$ and $w$. In a word, the endpoints of edges with cover level $c_{l}(e)=-1$ should be reserved in $\mathcal{C}_{t}$.

By analogy to the case of edge deletion, we deal with the edge insertion in Lemma 3. When inserting an edge, we should first decrease some $\nu$ value, and then query whether or not a vertex $x$ satisfies $\nu(x)=1$. If the predecessor and successor of a vertex $x$ along $v \ldots w$ were not 2-edge connected in $\widetilde{\mathcal{D}}_{t}$ before inserting $(v, w)$, its $\nu(x)$ value need to be decreased, which indicates that $x$ is not covered. Hence, we should mark the endpoints of edges with cover level $c_{l}(e)=-1$ before inserting $(v, w)$ and then decrease the $\nu$ value of these endpoints. Based on the above discussions, the adaptive minimal $d$-CDS (AMDC) algorithm is presented as follows

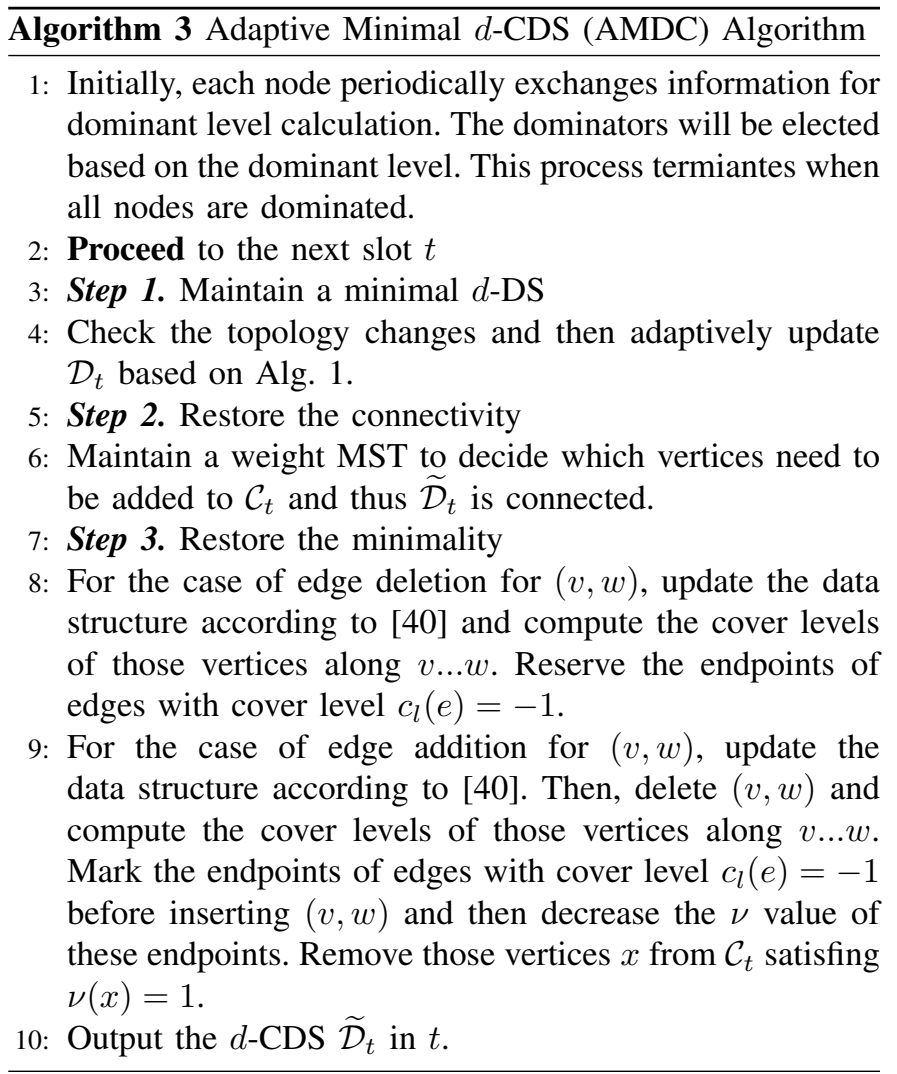

Finally, we prove some crucial properties about Alg. 3 .

Lemma 4: The Alg. 3 can dynamically restore the minimality of $\mathcal{C}_{t}$ and the connectivity of $\widetilde{\mathcal{D}}_{t}$ in each $t$.

Proof: By dynamically maintaining a weight MST of $\widetilde{\mathcal{D}}_{t}$ in response to every types of topology changes, the Alg. 3 can restore its connectivity. Besides, the step 3 in Alg. 3 can ensure that all reserved vertices in $\mathcal{C}_{t}$ are articulation points in $\widetilde{\mathcal{D}}_{t}$, which proves the Lemma 4 .

Lemma 5: The Alg. 3 has an approximation ratio $\mathcal{O}(d \log (N))$ for minimal $d$-CDS. 
Proof: To prove this lemma, we first construct a graph $G_{p}^{t}=$ $(X, F)$, in which vertex set $X$ are either in $\mathcal{C}_{t}$ or the connected components of $\mathcal{D}_{t}$. The edge set $F$ can be built as follows.

First, we arbitrary select one vertex from each connected component of $\mathcal{D}_{t}$ and then insert vertices from $\mathcal{C}_{t}$ one by one. For an inserted vertex $v$, we should match it with a vertex $u \in \mathcal{D}_{t}$ or $u \in \mathcal{C}_{t}$ adjacent to it to ensure that $(u, v)$ cannot connect $F$ into a cycle. Then, we insert $(u, v)$ into $F$ and ignore other vertices in the same connected component containing $u$. Finally, this process generates a spanning forest where each vertex of $\mathcal{C}_{t}$ is either adjacent to a connected component of $\mathcal{D}_{t}$ or another vertex in $\mathcal{C}_{t}$. Considering that $\mathcal{C}_{t} \cup \mathcal{D}_{t}$ is connected, we can add some edges to make $F$ a tree. For this tree, the leaves are vertices corresponding to connected component. Otherwise, those leaves belonging to $\mathcal{C}_{t}$ can be removed without losing the connectivity, which contradicts the minimality. Besides, in the construction process of $F$, each vertex of $\mathcal{C}_{t}$ must be adjacent to a vertex corresponding to a connected component of $\mathcal{D}_{t}$ or another vertex in $\mathcal{C}_{t}$ whose shortest distance from a connected component of $\mathcal{D}_{t}$ is at most $d$-hop. The above two conditions can guarantee that for each subtree with any vertex in $\mathcal{C}_{t}$, there exists a vertex in $\mathcal{D}_{t}$ whose minimal distance from this root node is at most $2 d$. Otherwise, some vertices are not $d$-hop dominated. Considering that any vertex of $\mathcal{C}_{t}$ is not a leaf node, arbitrarily rooting the tree on a vertex in $\mathcal{C}_{t}$, and then every vertex of $\mathcal{C}_{t}$ has a descendant from $\mathcal{D}_{t}$ within distance $2 d$. In other word, at lease one vertex belongs to $\mathcal{D}_{t}$ in any connected $2 d+1$ vertices, which indicates that $\left|\mathcal{C}_{t}\right| \leq 2 d\left|\mathcal{D}_{t}\right|$. Hence, $\widetilde{\mathcal{D}}_{t}=\mathcal{O}(d \log (N))$. Opt.

Lemma 6: The amortized time complexity of Alg. 3 can be bounded by $\widetilde{\mathcal{O}}\left(d \Delta^{2}\right)$ per update.

Proof: We analyze the complexity step by step. Firstly, the complexity of step 1 in Alg. 3 is $\widetilde{\mathcal{O}}\left(d \Delta^{2}\right)$ by invoking the Alg. 1. As discussed before, the cost of vertex insertion/delection from $\widetilde{\mathcal{D}}_{t}$ is also $\widetilde{\mathcal{O}}(\Delta)$ and the number of changes can be bounded by $d \Delta$ caused by vertex deletion from $\mathcal{C}_{t}$, and thus the complexity of step 2 is $\widetilde{\mathcal{O}}\left(d \Delta^{2}\right)$. For step 3 , the cost of detecting articulation points is $\widetilde{\mathcal{O}}(1)$ for each added/removed edge and the cost for inserting/deleting a vertex can be bounded by $\widetilde{\mathcal{O}}(\Delta)$. In sum, the Alg. 3 takes $\widetilde{\mathcal{O}}\left(d \Delta^{2}\right)$ amortized time per update.

\section{Simulation Results}

To validate our theoretical analysis, we evaluate the proposed AMMCDS from several performances including the routing overhead, response time, and maintenance cost. Some basic simulation parameters are set according to some existing works [31], [38], [39], which are widely used for modeling the mobility, communication, and power system of FANETs. Some essential parameters are summarized in Table I unless otherwise noted.

Besides, some parameters about UAVs such as the angle of attack can be referred to the drone Matrice 100 produced by DJI [38]. For the communication radius, we directly set $R_{c}$ by simplifying other parameters in E.q. (4) based on different simulation environments, i.e., $R_{c}$ is set to a fixed value $120 \mathrm{~m}$ in homogeneous scenarios and within a range $[90,120]$ for different UAVs in heterogeneous scenarios. Similar to [15],
TABLE I: Main Simulation Parameters

\begin{tabular}{|c|c|}
\hline Parameters & Value \\
\hline Network area & $\left(3 \times 3 \times 3 \mathrm{~km}^{3}\right)$ \\
\hline Number of UAVs & A varying variable $N$ \\
\hline Number of time slot and duration & $5000,2 \mathrm{~s}$ \\
\hline Maximum speed of UAVs & $20 \mathrm{~m} / \mathrm{s}$ \\
\hline Battery capacity & $2 \times 10^{5} \mathrm{~J}$ \\
\hline Adjustable coefficient $\epsilon$ & 1 \\
\hline Unstable distance $R_{u}$ & $20 \mathrm{~m}$ \\
\hline Safe distance $R_{s}$ & $140 \mathrm{~m}$ \\
\hline
\end{tabular}

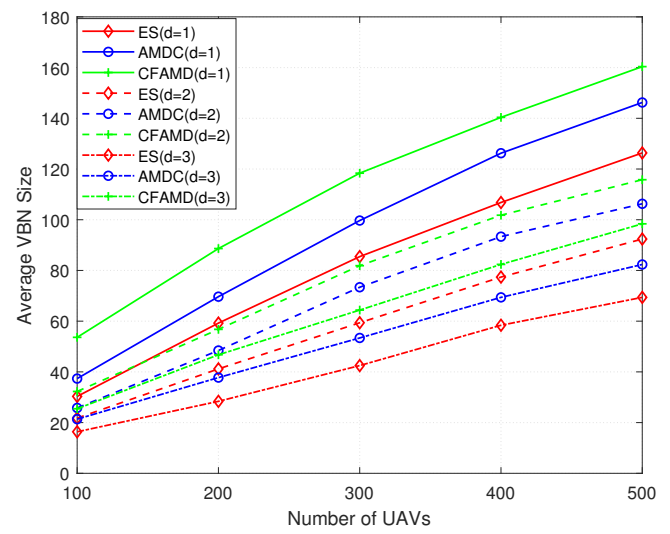

Fig. 4: Average VBN size in heterogeneous scenarios w.r.t $N$.

we only focus on the performance evaluation on fixed hop setting $(d=1,2$, and 3$)$, since $d>3$ may cause unacceptable path delay [11].

Furthermore, we compare our proposed algorithm with different benchmark algorithms. Firstly, we compare AMDC with a constant-factor approximation minimal $d$-CDS (CFAMD) algorithm in [15] with time complexity $\mathcal{O}\left(N^{2}\right)$ for each update. Moreover, "Exhaustive Search (ES)" can achieve the performance upper bound with extremely high complexity. It is worth mentioning that the approximation ratio of the CFAMD is only effective in geometric graphs. While in general graphs, the CFAMD is degraded to a heuristic algorithm since the theoretical analysis is invalid. To verify that our solution can adapt to all kinds of graphs, we evaluate the routing overhead and maintenance cost on both heterogeneous scenarios (abstracted as general graphs) and homogeneous scenarios (converted to geometric graphs), respectively. And evaluating the response time on homogeneous scenarios, because this metric is not related to graph structures. All these algorithms are implemented in Matlab R2019b and run on Dell Computer Inspiron5488 installed Windows 10 with CPU Intel Core i7 at $1.80 \mathrm{GHz}$ and RAM $8 \mathrm{~GB}$.

Fig. 4 and Fig. 5 show the average VBN size with varying number of UAVs in different scenarios. In homogeneous scenarios, the average VBN size attained by our proposed AMDS is smaller than that of CFAMD with $(d+1) \beta$-approximation ratio where $\beta=12$ for $d=1,125$ for $d=2$, and $12+\left(8 d^{3}+12 d^{2}+6 d\right) /\lceil 0.5\lfloor 0.5(d-1)\rfloor\rceil$ for $d=3$. The reason is that when $N$ is not large our solution always has a better approximation ratio since the $\log (N)$ term is rising slowly. In heterogeneous scenarios where the approximation ratio of CFAMD is invalid, i.e., the CFAMD is degraded to a heuristic 


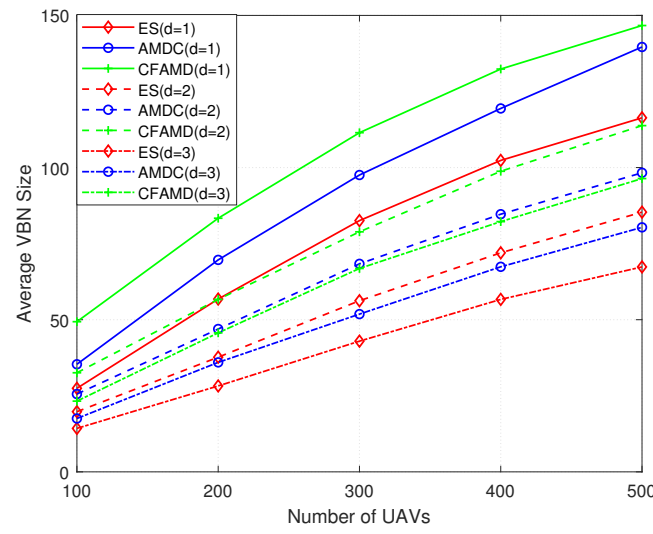

Fig. 5: Average VBN size in homogeneous scenarios w.r.t $N$.

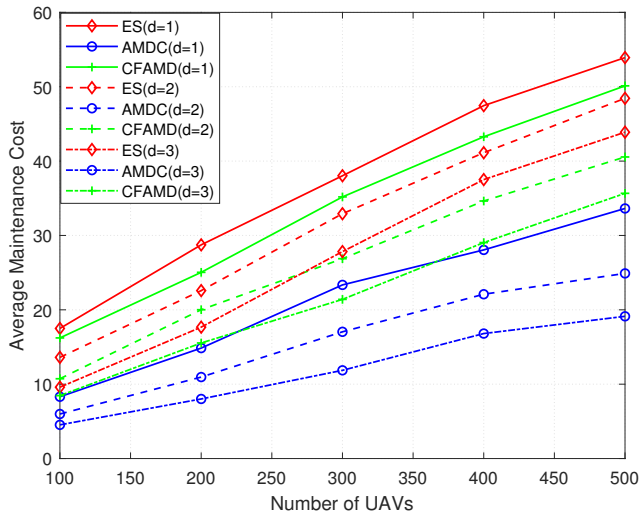

Fig. 6: Average maintenance cost in heterogeneous scenarios w.r.t $N$.

algorithm, our solution still has a better performance. From the perspective of algorithm design, the AMDS adaptively restores the minimality of $\mathcal{C}_{t}$ by removing the invalid articulation points whose removal has not impact on the connectivity of $\widetilde{\mathcal{D}}_{t}$ while the CFAMD cannot remove all such redundant vertices. It is obvious that the performance gap between AMDC and CFAMD for the same $d$ is narrowed with the increasing $N$. Meanwhile, with the increase of $d$, this gap becomes harder to narrow.

Fig. 6 and Fig. 7 shows the average maintenance cost with varying number of UAVs in different scenarios, which can be calculated by E.q. (20). Based on these two figures, we can observe that AMDS has the minimal maintenance cost. Because the solution in $t$ is based on that in $t-1$ and the topology changes $T C_{t-1}^{t}$ from $t-1$ to $t$ rather than selecting from scratch. In this way, AMDS can avoid extensive recomputing, which naturally reduces the maintenance cost. Besides, both ES and CFAMD ignor some important metrics such as connection duration and residual energy when selecting $d$-DS, which causes more frequent topology changes. The Fig. 8 further demonstrate the advantage of avoiding recomputing the solution from scratch. This advantage significantly saves time to deal with each element addition/removal. Besides, the performance gap between AMDS and CFAMD gradually increases with respect to $N$, this is not only because of the topology change frequency, but also because the time

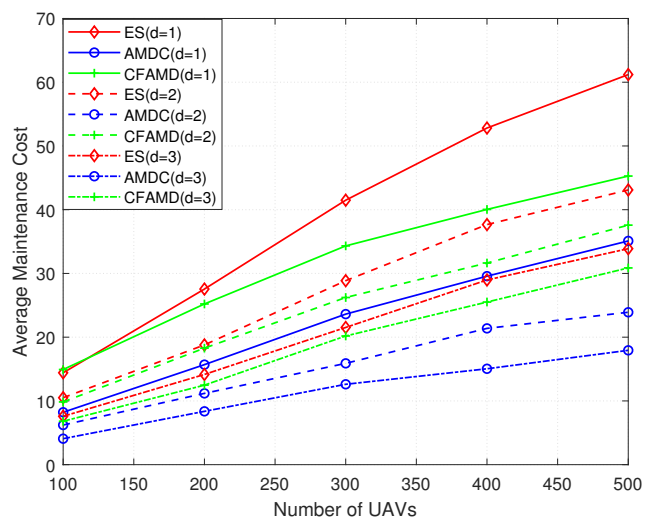

Fig. 7: Average maintenance cost in homogeneous scenarios w.r.t $N$.

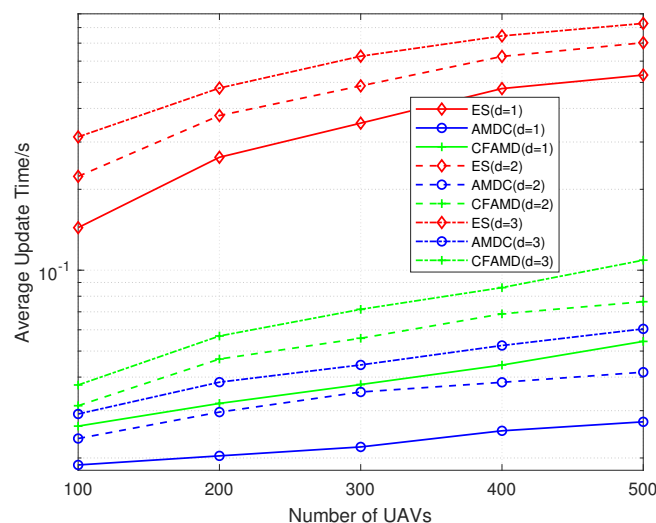

Fig. 8: Average update time w.r.t $N$.

complexity $\mathcal{O}\left(N^{2}\right)$ of CFAMD for each update grows faster than that of AMDS as $N$ increases.

According to the above comparison and analysis, AMDS can achieve the better update time and maintenance cost performances compared with the exact optimal solution by sacrificing part of the routing overhead performance. The reason is that AMDS fails to provide sufficiently strong optimality guarantees. However, according to the simulation results compared with other state-of-the-art distributed approximation algorithm, AMDS is dominant in all three performance indicators. The only drawback is flexibility since AMDS is mixed distributed/centralized algorithm. In sum, AMDS can achieve the better trade-off among the routing overhead, update time, and maintenance cost in both heterogeneous scenarios and homogeneous scenarios. In addition, its flexibility is between centralized optimal solution and fully distributed algorithm. Most importantly, our solution can adapt to all kinds of topology changes and the performance bound is always effective in different graphs. Besides, another trade-off here is that as we reduce routing cost (VBN size) and maintenance cost by increasing $d$, the time complexity and path delay (the average path length increase with maximal hop count $d$ ) can increase. Hence, $d$ can be flexibly set based on real scenarios.

\section{CONCLUSION}

In this paper, we developed an adaptive solution framework for solving the stable VBN maintenance problem in response 
to any types of topology changes in FANETs. More specifically, we proposed an AMDS algorithm with approximation ratio $\mathcal{O}(d \log (N))$ and expected $\widetilde{\mathcal{O}}\left(d \Delta^{2}\right)$ time complexity per update. Simulation results show that our proposed algorithm can strike a better trade-off among the routing overhead, update time, and maintenance costs per topology update compared with state-of-the-art $d$-CDS scheme in dynamic environments. We believe that the adaptive solution framework presented here can be applicable to other dynamic topology control problem of FANETs in emergency scenarios, such as adaptive multihop link reconstruction.

\section{REFERENCES}

[1] O. K. Sahingoz, "Mobile networking with UAVs: Opportunities and challenges," in Proc. IEEE ICUAS'13, (Atlanta, GA, USA), pp. 933 941, May. 2013.

[2] E. Frew and T. Brown, "Networking issues for small unmanned aircraft systems," J. Intell. Robot. Syst., vol. 54, pp. 21-37, Mar. 2009.

[3] D. Wu, R. Bao, Z. Li, H. Wang, H. Zhang, and R. Wang, "Edge-cloud collaboration enabled video service enhancement: A hybrid humanartificial intelligence scheme," IEEE Trans. Multimedia, pp. 1-13(Early Access), Mar. 2021

[4] D. Wu, Z. Yang, B. Yang, R. Wang, and P. Zhang, "From centralized management to edge collaboration: A privacy-preserving task assignment framework for mobile crowdsensing," IEEE Internet Things J., vol. 8, pp. 4579-4589, Mar. 2021.

[5] O. Sahingoz, "Networking models in flying ad-hoc networks (FANETs): Concepts and challenges," J. Intell. Robot. Syst., vol. 74, Apr. 2014.

[6] I. Bekmezci, O. K. Sahingoz, and S. Temel, "Flying ad-hoc networks (FANETs): A survey," Ad Hoc Netw., vol. 11, pp. 1254-1270, May. 2013.

[7] L. D. Nguyen, A. Kortun, and T. Q. Duong, "An introduction of real-time embedded optimisation programming for UAV systems under disaster communication," EAI Endorsed Trans. Indust. Netw. \& Intellig. Syst., vol. 5, p. e5, Dec. 2018.

[8] B. Wang, Y. Sun, D. Liu, H. M. Nguyen, and T. Q. Duong, "Socialaware UAV-assisted mobile crowd sensing in stochastic and dynamic environments for disaster relief networks," IEEE Trans. Vehi. Tech., vol. 69, pp. 1070-1074, Jan. 2020.

[9] S. Rosati, K. Krużelecki, G. Heitz, D. Floreano, and B. Rimoldi, "Dynamic routing for flying ad hoc networks," IEEE Trans. Vehicul. Tech., vol. 65, pp. 1690-1700, Mar. 2016.

[10] J. Akbari Torkestani, "A stable virtual backbone for wireless MANETS," Telecommun. Syst., vol. 55, pp. 137-148, Jan. 2014.

[11] B. Das and V. Bharghavan, "Routing in ad-hoc networks using minimum connected dominating sets," in Proc. IEEE ICC'97, vol. 1, (Montreal, Quebec, Canada), pp. 376-380, Jun. 1997.

[12] E. Sampathkumar and H. Walikar, "The connected domination number of a graph," J. Math. Phys., vol. 13, p. 607-613, Jan. 1979.

[13] R. J. Wilson, Introduction to Graph Theory. New York, NY, USA: John Wiley \& Sons, Inc., 1986.

[14] D. Ding-Zhu and W. Peng-Jun, Connected dominating set: theory and applications. New York, NY, USA: Springer Science \& Business Media, 2012.

[15] K. Li, X. Gao, F. Wu, and G. Chen, "A constant factor approximation for d-hop connected dominating set in three-dimensional wireless networks," IEEE Trans. Wireless Commun., vol. 18, pp. 4357-4367, Jul. 2019.

[16] O. S. Oubbati, A. Lakas, P. Lorenz, M. Atiquzzaman, and A. Jamalipour, "Leveraging communicating UAVs for emergency vehicle guidance in urban areas," IEEE Trans. Emerg. Top. Comput., pp. 1-13 (Early Access), 072019

[17] K. Tao, X. Sun, K. Zhang, and L. Liu, "Distributed construction of faulttolerance virtual backbone network for UAV cluster network," in Proc. EAI ADHIP'17, (Harbin, China), pp. 226-233, 2017.

[18] Y. Li, M. T. Thai, F. Wang, C.-W. Yi, P.-J. Wan, and D.-Z. Du, "On greedy construction of connected dominating sets in wireless networks: Research articles," Wirel. Commun. Mob. Comput., vol. 5, pp. 927-932, Dec. 2005.

[19] D. fu, L. Han, Z. Yang, and S. Jhang, "A greedy algorithm on constructing the minimum connected dominating set in wireless network," Int. $J$. of Distrib. Sens. Netw., vol. 12, pp. 1703201-1703201, Jul. 2016.
[20] J. P. Mohanty, C. Mandal, C. Reade, and A. Das, "Construction of minimum connected dominating set in wireless sensor networks using pseudo dominating set," Ad Hoc Netw., vol. 42, pp. 61-73, 2016.

[21] M. Guruprakash and B. Mallikarjunaswamy, "Algorithmic aspects for total connected dominating sets in mobile ad hoc wireless networks," Int. J. Inform. Tech. Comput. Sci., vol. 4, pp. 34-40, Mar. 2012.

[22] T. H. P. Vuong and D. T. Huynh, "Adapting d-hop dominating sets to topology changes in ad hoc networks," in Proc. IEEE ICCCN, pp. 348353, Oct. 2000.

[23] M. Azizian, S. Cherkaoui, and A. S. Hafid, "A distributed d-hop cluster formation for vanet," in Proc. IEEE WCNC, (Doha, Qatar), pp. 1-6, Apr. 2016.

[24] Z. Zhang, Q. Liu, and D. Li, "Two algorithms for connected r-hop kdominating set," Discrete Math. Algorithms Appl., vol. 1, pp. 485-498, Dec. 2009.

[25] X. Gao, X. Zhu, J. Li, F. Wu, G. Chen, D. Du, and S. Tang, "A novel approximation for multi-hop connected clustering problem in wireless networks," IEEE/ACM Trans. Netw., vol. 25, no. 4, pp. 2223-2234, 2017.

[26] A. K. A. Gupta, R. Krishnaswamy and D. Panigrahi., "Online and dynamic algorithms for set cover," in Proc. ACM STOC'17, (Montreal, Canada), pp. 537-550, Jun. 2017.

[27] X. Li, T. Pan, M. A. Alim, and M. T. Thai, "Resource allocation in highly dynamic device-to-device communication: An adaptive set multi-cover approach," IEEE Trans. Wireless Commun., vol. 18, pp. 4607-4619, Oct. 2019.

[28] B. Wang, Y. Sun, T. Q. Duong, L. D. Nguyen, and L. Hanzo, "Riskaware identification of highly suspected COVID-19 cases in social IoT: A joint graph theory and reinforcement learning approach," IEEE Access, vol. 8, pp. 115655-115661, Jun. 2020.

[29] B. Wang, Y. Sun, Z. Sun, L. D. Nguyen, and T. Q. Duong, "UAV-assisted emergency communications in social IoT: A dynamic hypergraph coloring approach," IEEE Internet Things J., vol. 7, pp. 7663-7677, Aug. 2020.

[30] M. Gupta and S. Khan, "Simple dynamic algorithms for maximal independent set and other problems," arXiv, 2018.

[31] X. Qi, X. Gu, Q. Zhang, and Z. Yang, "A link-estimation based multiCDSs scheduling mechanism for FANET topology maintenance," in Proc. EAI SINC'19, (Wuzhen, China), pp. 66-86, Sep. 2019.

[32] L. Guibas, N. Milosavljević, and A. Motskin, "Connected dominating sets on dynamic geometric graphs," Comput. Geom. Theory Appl., vol. 46, pp. 160-172, Feb. 2013.

[33] N. Hjuler, G. F. Italiano, N. Parotsidis, and D. Saulpic, "Dominating sets and connected dominating sets in dynamic graphs," arXiv e-prints, 2019.

[34] N. Wu, C. Chacon, Z. Hakl, K. Petty, and D. Smith, "Design and implementation of an unmanned aerial and ground vehicle recharging system," in IEEE NAECON'19, (Dayton, OH, USA), pp. 163-168, Jul. 2019.

[35] X. Lin, V. Yajnanarayana, S. D. Muruganathan, S. Gao, H. Asplund, H. Maattanen, M. Bergstrom, S. Euler, and Y. P. E. Wang, "The sky is not the limit: LTE for unmanned aerial vehicles," IEEE Commun. Mag., vol. 56, pp. 204-210, Apr. 2018.

[36] D. Shumeye Lakew, U. Sa'ad, N. Dao, W. Na, and S. Cho, "Routing in flying ad hoc networks: A comprehensive survey," IEEE Commun. Surv. Tut., vol. 22, no. 2, pp. 1071-1120, 2020.

[37] C. Reynolds, "Flocks, herds, and schools: A distributed behavioral model," ACM SIGGRAPH Comput. Graph., vol. 21, pp. 25-34, 071987.

[38] X. Li, H. Yao, J. Wang, X. Xu, C. Jiang, and L. Hanzo, "A near-optimal UAV-aided radio coverage strategy for dense urban areas," IEEE Trans. Vehicul. Tech., vol. 68, pp. 9098-9109, Jul. 2019.

[39] Z. Liu, R. Sengupta, and A. Kurzhanskiy, "A power consumption model for multi-rotor small unmanned aircraft systems," in Proc. IEEE ICUAS'17, (Miami, FL, USA), pp. 310-315, 2017.

[40] J. Holm, K. de Lichtenberg, and M. Thorup, "Poly-logarithmic deterministic fully-dynamic algorithms for connectivity, minimum spanning tree, 2-edge, and biconnectivity," J. ACM, vol. 48, pp. 723-760, Jul. 2001. 


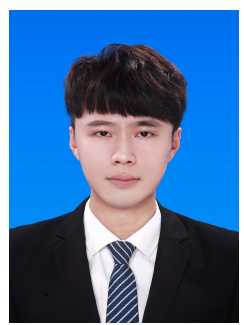

Bowen Wang received the Ph.D. degree in information and communication engineering from China University of Mining and Technology, Xuzhou, China, in 2020. He was also a visiting Ph.D student at the School of Electronics, Electrical Engineering and Computer Science, Queen's University Belfast from 2018 to 2019. He is currently an Associate Professor at the School of Information and Control Engineering, China University of Mining and Technology. He was awarded the best paper award in INISCOM 2019. His research interests include matching theory, graph theory, UAV communications, and Social Internet of Things (SIoT).

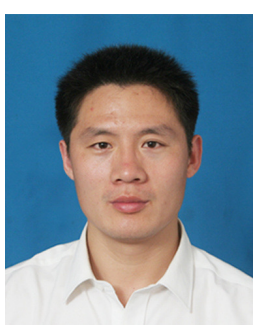

Yanjing Sun (M'16) received the Ph.D. degree in information and communication engineering from China University of Mining and Technology, Xuzhou, China, in 2008. He is currently a Professor at the School of Information and Control Engineering and the director of Network Information Center, China University of Mining and Technology. His current research interests include IBFD communications, embedded real-time system, wireless sensor networks and cyber-physical systems.

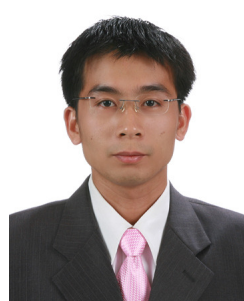

Trung Q. Duong (S'05, M'12, SM'13)is a Professor and Chair of Telecommunications at Queen's University Belfast (UK), where he was a Lecturer (Assistant Professor) (2013-2017), a Reader (Associate Professor) (2018-2020), and Full Professor from August 2020. He also holds a prestigious Research Chair of Royal Academy of Engineering. His current research interests include wireless communications, machine learning, realtime optimisation, and data analytic. He is the author or co-author of over $350+$ technical papers published in scientific journals (230+ articles) and presented at international conferences (140+ papers).

Dr. Duong currently serves as an Editor for the IEEE TRANSACTIONS ON WIRELESS COMMUNICATIONS and an Executive Editor for IEEE COMMUNICATIONS LetTERs. He was awarded the Best Paper Award at the IEEE Vehicular Technology Conference (VTC-Spring) in 2013, IEEE International Conference on Communications (ICC) 2014, IEEE Global Communications Conference (GLOBECOM) 2016 and 2019, IEEE Digital Signal Processing Conference (DSP) 2017, and International Wireless Communications \& Mobile Computing Conference (IWCMC) 2019. He is the recipient of prestigious Royal Academy of Engineering Research Fellowship (2015-2020) and has won a prestigious Newton Prize 2017.

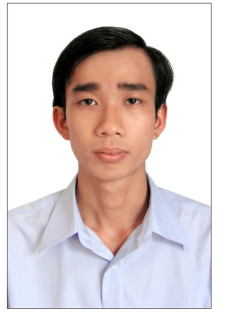

Tan Do-Duy received his B.S. degree from Ho Chi Minh City University of Technology (HCMUT), Vietnam, and M.S. degree from Kumoh National Institute of Technology, Korea, in 2010 and 2013, respectively. He received his Ph.D. degree from Autonomous University of Barcelona, Spain, in 2019. He is currently with the Department of Computer and Communication Engineering, Ho Chi Minh City University of Technology and Education (HCMUTE) in Vietnam as an Assistant Professor. His main research interests include real-time optimisation for resource allocation in wireless networks and coding applications for wireless communications.

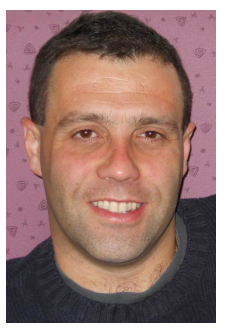

Emiliano Garcia-Palacios is a senior lecturer at Queen's University Belfast. He received a Ph.D. from Queen's University Belfast in 2000 and since then has been leading research in wireless network resource management. His research interests include wireless protocols, wireless resource allocation, and optimization and traffic management for next generation gigabit networks. 